\title{
Polarimetric Passive Radar: a Practical Approach to Parametric Adaptive Detection
}

\author{
Francesca Filippini, Member, IEEE, and Fabiola Colone, Senior Member, IEEE
}

\begin{abstract}
Polarimetric diversity has been recently shown to significantly improve the target detection performance in passive radar systems, if properly exploited to mitigate the competing interference. An adaptive processing scheme is presented in this work, leveraging the information conveyed using multi-polarized receiving antennas and modeling the disturbance as a multichannel autoregressive process. Despite this approach operates with a limited number of adaptive degrees of freedom, the long integration time exploited by passive radar typically requires a substantial computational cost and ad hoc expedients for its application. Therefore, a modified cost-effective implementation of the conceived solution is proposed in order to reduce the computational burden while controlling the resulting loss. The authors extensively demonstrate the effectiveness of the proposed solution against experimental data collected by a FM radio based passive coherent location system. The experimental results show that the proposed processing scheme yields a remarkable improvement with respect to both the conventional processing at the single polarimetric channel and the state-ofthe-art strategies exploiting polarization diversity in PCL systems.
\end{abstract}

Index Terms - passive radar, polarization diversity, multichannel autoregressive model, FM radio signal, adaptive target detection

\section{INTRODUCTION}

$\mathrm{P}$ ASSIVE bistatic radar (PBR), also known as passive coherent location (PCL), technology has been attracting significant research interests over the past two decades [1][2][3][4]. The wide interest received by PCL sensors allowed them to increasingly reach a point of maturity. Nevertheless, by relying on signals emitted by illuminators of opportunity (IOs) to detect and localize targets, the performance of PCL sensors might still be strongly limited. The main limitations stem from the lack of control over the exploited waveform structure as well as from the strong direct signal and multipath contributions. In addition, significant interference can be experienced due to co-channel or adjacentchannel transmissions, especially when broadcast emitters are exploited as IOs.

F. Filippini and F. Colone are with the Department of Information Engineering, Electronics and Telecommunications (DIET), Sapienza University of Rome, 00184 Rome, Italy.

e-mail: francesca.filippini@uniroma1.it, fabiola.colone@uniroma1.it. Corresponding author: Francesca Filippini.
Recently, among the advanced processing strategies devised to overcome these limitations, the exploitation of polarimetric diversity has been considered [5]-[21]. In [5][17], the target detection stage is addressed, observing that target echoes typically show a random polarization and therefore the use of a fixed polarization on receive might result in a significant signal to noise ratio (SNR) degradation. Consequently, it is expected that a combination of signals received via differently polarized antennas yields a detection performance improvement, provided that proper strategies are employed. Different solutions along this line have been proposed and have been shown to effectively increase the PCL system reliability against those effects that are not under the control of the radar designer. These advantages have been demonstrated against different operative geometries as well as exploiting different sources of opportunities.

A first attempt toward this direction is represented by a simple non-coherent integration (NCI) of the range-Doppler maps obtained at multi-polarized surveillance channels [5][6][7]. The polarimetric NCI strategy aims at increasing the SNR of the target echo, however it does not fruitfully exploit the information diversity to reject the disturbance contributions, such as cancellation residuals or interfering signals. Therefore, the authors in [8] have proposed and investigated a more effective polarimetric locally adaptive detection scheme based on a generalized likelihood ratio test (GLRT) applied over the range-Doppler domain. The latter strategy adaptively exploits the polarimetric information to discriminate targets from the competing disturbance, thus significantly improving the target detection performance of the system. The effectiveness of this approach has been extensively demonstrated using different IOs and operative scenarios, showing its capability to successfully mitigate the disturbance contributions [8]-[10].

An alternative and efficient solution was investigated in [11], where the authors considered the possibility of globally adapting the filter weights of the polarimetric GLRT in the temporal domain. Both the approaches in [8] and [11] operate adaptively in the polarimetric domain only. However, based on the comparison reported in [8], the local estimation of the filter weights performed over the range-Doppler domain is shown to be more robust to the spectral characteristics of the disturbance. The latter consideration suggests the possibility to adaptively jointly exploit the polarimetric and temporal domain to better counteract the disturbance contributions, thus further improving the system performance. 
To this purpose, we leverage our recent works [15][16] where we presented a novel parametric adaptive detection scheme, devised for a generic multipolarimetric radar system. Specifically, the proposed detection strategy is derived by modeling the disturbance as a multichannel autoregressive (AR) process and by resorting to a two-stage GLRT approach. The advantages of this detection scheme, referred to as modified polarimetric autoregressive model based adaptive matched filter (Mod-Pol-AR-AMF), have been extensively demonstrated both against simulated and experimental data collected via a polarimetric active radar system [16]. The same approach has been preliminarily applied to the case of a PCL system in [16][17]. In those works, along with some prelimary promising results, also some critical matters have been identified, arising from the direct application of the Mod-PolAR-AMF to the case of a PCL system. The most critical issues stem from the use of long coherent processing intervals (CPIs), typically required for PCL systems to achieve reasonable SNR values. This requirement on the one hand, severely affects the computational complexity of the considered processing and, on the other hand, jeopardizes some assumptions underlying the adaptive implementation of the proposed approach.

In the present paper we build upon the promising results obtained in [15][16][17], and we present a new computationally efficient version of the Mod-Pol-AR-AMF, specifically devised for PCL systems. In detail, different strategies are presented and compared to adapt and update the filter weights. Moreover, appropriate approximations are adopted to reduce the computational complexity of the original algorithm while limiting the corresponding loss, as validated via a theoretical analysis of the achievable performance. These modifications enable an extensive validation to be carried out, using both simulated and real data. For the experimental validation, we exploit a dataset collected by a multi-channel FM radio-based PCL system, equipped with two linearly polarized reference antennas and two linearly polarized surveillance antennas. We demonstrate the benefits of the proposed solution with respect to the conventional PCL processing performed at the single polarimetric channel as well as with respect to state-of-the-art approaches. Furthermore, we provide useful considerations for it to be applied in different operative scenarios exploiting different IOs.

The remainder of the paper is organized as follows. In Section II, the AR model based polarimetric adaptive strategy is presented and adapted to the PCL case. Section III introduces the approximation to be adopted in order to reduce the computational burden while ensuring negligible loss. In Section IV the relevant parameters of the proposed approach are carefully selected by means of an extensive comparative analysis of the resulting performance. Finally, in Section V we compare the obtained performance with existing solutions while Section VI reports our concluding remarks.

\section{AR MODEL BASED POLARIMETRIC ADAPTIVE DETECTOR}

\section{A. Signal Model}

Let us consider a polarimetric PCL system that simultaneously collects target echoes from $L$ differently polarized receiving antennas connected to $L$ surveillance channels (see Fig.1). The number of polarimetric channels strictly depends on the considered application and on the employed IO. For instance, the most common configuration is represented by two orthogonal linearly polarized antennas collecting echoes at both the horizontal $(\mathrm{H})$ and vertical $(\mathrm{V})$ polarizations $(L=2)$. This is also the configuration employed in this work for an extensive validation. However, the following mathematical development is reported regardless of the specific number of available channels and does not rely on any assumption on $L$ for it to be exploited in future studies.

According to the polarimetric processing scheme introduced in [8], the $L$ surveillance signals first separately undergo the temporal disturbance cancellation stage. This stage is aimed at reducing the direct signal, clutter and multipath contributions received along with the target echoes and it relies on the availability of a good copy of the transmitted signal collected by a dedicated reference antenna. This temporal cancellation stage can be performed according to different solutions, for instance the Extensive Cancellation Algorithm (ECA) [22] or its polarimetric version (Pol-ECA) [8], if $R>1$ reference signals are made available using $R$ polarimetric reference channels. In this work, we use a PolECA with $R=2$ and we refer the interest reader to [8] for additional details. Once this stage has been performed, $L$ sequences of samples are available where the direct signal, clutter and multipath contributions have been strongly reduced. However, despite the effectiveness of the temporal disturbance cancellation stage, it is likely for the $L$ signals to include cancellation residuals and interfering contributions, along with target echoes.

We arrange the data samples from the available polarimetric surveillance channels at the $m$-th temporal observation, $m=0, \ldots, M-1$, in a $L$-dimensional vector $\mathbf{x}_{0}(m)$, as follows:

$$
\mathbf{x}_{0}(m)=\left[x_{0}^{(0)}(m) \ldots x_{0}^{(l)}(m) \ldots x_{0}^{(L-1)}(m)\right]^{T}
$$

denoting $M$ as the number of samples in the considered CPI, i.e. $M=\left\lfloor T_{\text {int }} f_{s}\right\rfloor$, where $T_{\text {int }}$ and $f_{s}$ are the coherent integration time and the sampling frequency, respectively. Furthermore, we arrange the $M$ consecutive samples in a $L M-$ dimensional vector $\mathbf{x}_{0}=\left[\begin{array}{lll}\mathbf{x}_{0}^{T}(0) & \mathbf{x}_{0}^{T}(1) \ldots \mathbf{x}_{0}^{T}(M-1)\end{array}\right]^{T}$.

Under the null (or target absent) hypothesis $H_{0}$, vector $\mathbf{x}_{0}$ is solely composed by disturbance contributions $\mathbf{d}$, such as thermal noise, interference from transmissions at co-/adjacent channels and residual clutter and multipath contributions. Conversely, under the alternate (or target present) hypothesis $H_{1}$, vector $\mathbf{x}_{0}$ is composed by both disturbance and a useful target component $\mathbf{s}$. 


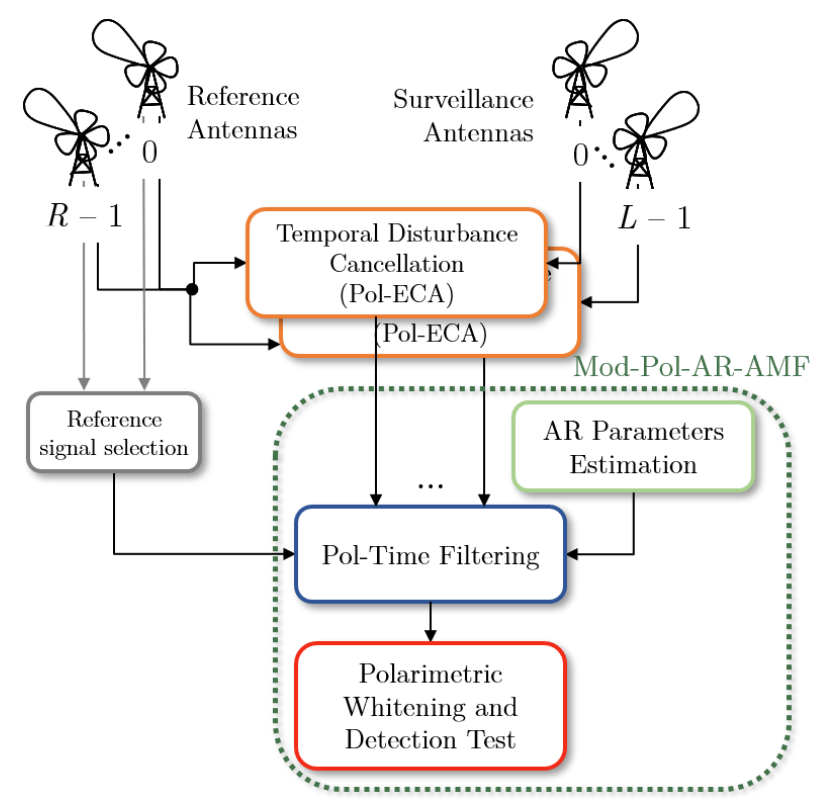

Fig.1 Multi-polarimetric PCL processing scheme.

Therefore, one can write:

$$
\begin{array}{ll}
H_{0}: & \mathbf{x}_{0}=\mathbf{d} \\
H_{1}: & \mathbf{x}_{0}=\mathbf{s}+\mathbf{d}
\end{array}
$$

The target component $\mathbf{s}$ can be written as:

$$
\mathbf{s}=\mathbf{t}\left(\tau, f_{D}\right) \otimes \boldsymbol{\alpha}
$$

denoting $\otimes$ as the Kronecker product, where

- $\mathbf{t}\left(\tau, f_{D}\right)$ is a $M$-samples fragment of the transmitted waveform $r(t)$, delayed in time by $\tau$ and Doppler shifted by $f_{D}$, i.e.

$$
\mathbf{t}\left(\tau, f_{D}\right)=\left[\begin{array}{c}
r\left(t_{0}-\tau\right) \\
r\left(t_{0}+\frac{1}{f_{s}}-\tau\right) \\
\vdots \\
r\left(t_{0}+\frac{M-1}{f_{s}}-\tau\right)
\end{array}\right] \odot\left[\begin{array}{c}
1 \\
e^{j 2 \pi \frac{f_{D}}{f_{s}}} \\
\vdots \\
j 2 \pi(M-1) \frac{f_{D}}{f_{s}}
\end{array}\right]
$$

being $t_{0}$ the CPI starting time and denoting $\odot$ as the Hadamard product;

- $\quad \boldsymbol{\alpha}=\left[\alpha_{0}, \ldots, \alpha_{L-1}\right]^{T}$ contains the unknown complex target amplitudes at the different polarimetric channels, assumed constant during the CPI.

As in [15], we model the disturbance as a $L$-channel AR process of order $(Q-1)$, denoted as $\operatorname{AR}(Q-1)$. Accordingly, the $L \times 1$ vector random process $\mathbf{d}(m)$ extracted at the $m$-th time lag satisfies the following relation

$$
\mathbf{d}(m)=\sum_{q=1}^{Q-1} \mathbf{A}^{H}(q) \mathbf{d}(m-q)+\boldsymbol{\varepsilon}(m)
$$

where $\{\mathbf{A}(q)\}_{q=1}^{Q-1}$ are complex-valued $L \times L$ matrix parameters encoding the regression coefficients at different polarimetric channels and $\boldsymbol{\varepsilon}(m) \sim \mathcal{C N}(\mathbf{0}, \mathbf{R})$ is the driving white noise sequence, denoting $\mathbf{R}$ as the $L \times L$ polarimetric covariance matrix.

Based on the considered model, the approximate (actually conditional) likelihood function of the data $\mathbf{x}_{0}$ under the $H_{\gamma}(\gamma$ $=0,1)$ hypothesis is written as

$$
\begin{aligned}
& f_{\gamma}\left(\mathbf{x}_{0} \mid \gamma \boldsymbol{\alpha}, \mathbf{R}, \mathbf{A}\right)=\left(\pi^{L}|\mathbf{R}|\right)^{-(M-Q+1)} . \\
& \exp \left\{-\sum_{m=1}^{M-Q+1}\left[\tilde{\mathbf{x}}_{0}(m)-\gamma \widetilde{\mathbf{s}}(m)\right]^{H} \mathbf{P}\left[\tilde{\mathbf{x}}_{0}(m)-\gamma \widetilde{\mathbf{s}}(m)\right]\right\}
\end{aligned}
$$

where $\tilde{\mathbf{x}}_{0}(m)$ and $\widetilde{\mathbf{s}}(m)$ are $Q L$-dimensional sub-vectors of $\mathbf{x}_{0}$ and $\mathbf{s}$, respectively, starting from the $m$-th elements, i.e. $\tilde{\mathbf{x}}_{0}(m)$ $=\left[\mathbf{x}_{0}^{T}(m) \ldots \mathbf{x}_{0}^{T}(m+Q-1)\right]^{T}$ and $\tilde{\mathbf{s}}(m)=\left[\mathbf{s}^{T}(m) \ldots \mathbf{s}^{T}(m+Q-1)\right]^{T}$. Moreover, $\mathbf{P}=\mathbf{H}^{H} \mathbf{R}^{-1} \mathbf{H}$, with $\mathbf{H}=\left[\begin{array}{ll}-\mathbf{A}^{H} & \mathbf{I}_{L}\end{array}\right]$ and $\mathbf{I}_{L}$ being the identity matrix.

\section{B. Polarimetric AR model based adaptive detector}

The sought polarimetric adaptive detector has been derived in [15] by resorting to a two-stage GLRT approach. First, the disturbance characteristics, namely matrices $\mathbf{A}$ and $\mathbf{R}$, are assumed known and the clairvoyant detector is derived; then, suitable maximum likelihood (ML) estimates of the AR parameters are plugged into the derived clairvoyant test. We refer the reader to [15] for further details on the derivation.

This approach is referred to as polarimetric AR model based adaptive matched filter (Pol-AR-AMF) and the resulting detection test to be applied at a given delay (range) Doppler cell under test (CUT) can be written as:

$$
\begin{gathered}
H_{1} \\
\mathbf{z}_{0}\left(\tau, f_{D}\right) \|^{2} \underset{H_{0}}{\gtrless} \varrho
\end{gathered}
$$

where $\varrho$ is the detection threshold and

$$
\mathbf{z}_{0}\left(\tau, f_{D}\right)=\mathbf{W}^{-\frac{H}{2}}\left(\tau, f_{D}\right) \sum_{m=0}^{M-Q} \boldsymbol{\Sigma}^{H}\left(m, \tau, f_{D}\right) \widehat{\mathbf{P}} \tilde{\mathbf{x}}_{0}(m)
$$

where the representation $(\cdot)^{-\frac{H}{2}}$ is a short notation for $\left[(\cdot)^{-\frac{1}{2}}\right]^{H}$,

$$
\mathbf{W}\left(\tau, f_{D}\right)=\frac{1}{2} \sum_{m=0}^{M-Q} \boldsymbol{\Sigma}^{H}\left(m, \tau, f_{D}\right) \widehat{\mathbf{P}} \boldsymbol{\Sigma}\left(m, \tau, f_{D}\right)
$$

and

$$
\boldsymbol{\Sigma}\left(m, \tau, f_{D}\right)=\tilde{\mathbf{t}}\left(m, \tau, f_{D}\right) \otimes \mathbf{I}_{L}
$$

being $\tilde{\mathbf{t}}\left(m, \tau, f_{D}\right)$ a $Q$-dimensional sub-vector of $\mathbf{t}\left(\tau, f_{D}\right)$ in (4), starting from the $m$-th sample, i.e. $\tilde{\mathbf{t}}\left(m, \tau, f_{D}\right)=$ $\left[t_{m}\left(\tau, f_{D}\right) \ldots t_{m+Q-1}\left(\tau, f_{D}\right)\right]^{T}$, being $v_{n}$ the $n$-th element of vector $\mathbf{v}$. 
Matrix $\widehat{\mathbf{P}}$ in (9) and (10) is evaluated by exploiting suitable estimates of matrices $\mathbf{A}$ and $\mathbf{R}$. To this purpose, the adaptive detection scheme relies on the availability of $K$ secondary data vectors $\mathbf{x}_{k}, k=1, \ldots, K$, assumed independent, identically distributed (i.i.d.), target free and sharing the same distribution of the primary data $\mathbf{x}_{0}$ under the $H_{0}$ hypothesis. Under these hypotheses, proper approximations of the ML estimates of A and $\mathbf{R}$ are readily obtained as in [23] and [15]:

$$
\widehat{\mathbf{A}}=\widehat{\mathbf{Q}}_{00}^{-1} \widehat{\mathbf{Q}}_{01}
$$

and

$$
\widehat{\mathbf{R}}=\frac{1}{K(M-Q+1)}\left(\widehat{\mathbf{Q}}_{11}-\widehat{\mathbf{Q}}_{01}^{H} \widehat{\mathbf{Q}}_{00}^{-1} \widehat{\mathbf{Q}}_{01}\right)
$$

where $\widehat{\mathbf{Q}}_{00}(L(Q-1) \times L(Q-1)), \widehat{\mathbf{Q}}_{01}(L(Q-1) \times L), \widehat{\mathbf{Q}}_{11}(L \times L)$ are blocks of the following matrix:

$$
\widehat{\mathbf{Q}}=\sum_{k=1}^{K} \sum_{m=0}^{M-Q} \tilde{\mathbf{x}}_{k}(m) \tilde{\mathbf{x}}_{k}^{H}(m)=\left[\begin{array}{ll}
\widehat{\mathbf{Q}}_{00} & \widehat{\mathbf{Q}}_{01} \\
\widehat{\mathbf{Q}}_{01}^{H} & \widehat{\mathbf{Q}}_{11}
\end{array}\right]
$$

We observe that the $L Q \times L Q$ matrix $\widehat{\mathbf{Q}}$ represents an estimate of the pol-time disturbance covariance matrix within a sub-CPI of length $Q$, being $Q$ the number of temporal degrees of freedom, namely taps, of the employed filter. As is apparent from (13), this estimate benefits from a joint average over $K$ secondary data and $M-Q+1$ consecutive (overlapped) sub-CPIs within the CPI.

The analyses in [15] have demonstrated the effectiveness of the Pol-AR-AMF against an input disturbance that exactly matches the employed $\operatorname{AR}(Q-1)$ model in (5). In contrast, we have demonstrated in [16] that when the spectral characteristics of the input disturbance differ from the employed AR model, some disturbance residual might arise after the temporal and polarimetric filtering. Such residual is encoded in the covariance matrix $\mathbf{D}_{z}\left(\tau, f_{D}\right)$ of the complex Gaussian random output vector $\mathbf{z}_{0}\left(\tau, f_{D}\right)$ that, differently from the case of a perfect spectral match, is not the identity matrix. Therefore, in order to make the detector robust with respect to typical spectral mismatches between the input disturbance and the employed model, an additional polarimetric whitening stage is included in the processing scheme based on a proper estimate of matrix $\mathbf{D}_{z}\left(\tau, f_{D}\right)$. The modified detection test proposed in [16] can be equivalently written as

$$
\begin{array}{r}
\mathbf{w}_{0}^{H}\left(\tau, f_{D}\right) \widehat{\mathbf{D}}_{w}^{-1}\left(\tau, f_{D}\right) \mathbf{w}_{0}\left(\tau, f_{D}\right) \stackrel{H_{1}}{\gtrless} \eta \\
H_{0}
\end{array}
$$

where $\eta$ is the detection threshold,

$$
\mathbf{w}_{0}\left(\tau, f_{D}\right)=\sum_{m=0}^{M-Q} \boldsymbol{\Sigma}^{H}\left(m, \tau, f_{D}\right) \widehat{\mathbf{P}} \widetilde{\mathbf{x}}_{0}(m)
$$

and $\widehat{\mathbf{D}}_{w}\left(\tau, f_{D}\right)$ is the sample covariance matrix of the filter output, namely $\mathbf{D}_{w}\left(\tau, f_{D}\right)=E\left\{\mathbf{w}_{0}\left(\tau, f_{D}\right) \mathbf{w}_{0}^{H}\left(\tau, f_{D}\right)\right\}=$ $\mathbf{W}^{-\frac{H}{2}}\left(\tau, f_{D}\right) \mathbf{D}_{z}\left(\tau, f_{D}\right) \mathbf{W}^{\frac{1}{2}}\left(\tau, f_{D}\right)$. It can be obtained using a set of $P$ training data, $\mathbf{w}_{p}(p=1, \ldots, P)$, that underwent the same filtering stages applied to the primary data, i.e. $\widehat{\mathbf{D}}_{w}\left(\tau, f_{D}\right)=$ $\frac{1}{P} \sum_{p=1}^{P} \mathbf{w}_{p} \mathbf{w}_{p}^{H}$. In this regard we recall that the secondary data to be exploited for the estimation of matrix $\mathbf{D}_{w}$ should not necessarily coincide with the secondary data exploited to build the first cancellation stage of the detector. For instance, an effective choice is to exploit the filter outputs at a set $I_{\left(\tau, f_{D}\right)}$ of $P$ indices that identify range-Doppler cells surrounding the CUT over the range-Doppler plane, i.e. $\mathbf{w}_{p}=\mathbf{w}_{0}\left(\tau_{p}, f_{D_{p}}\right)$, $\left(p \in I_{\left(\tau, f_{D}\right)},\left|I_{\left(\tau, f_{D}\right)}\right|=P\right)$.

In this work we use the detector in (14), referred to as modified Pol-AR-AMF (Mod-Pol-AR-AMF) for a PCL application and we show in Fig.1 a block diagram of the main signal processing stages foreseen in the considered multipolarimetric processing scheme.

An approximate analysis of the theoretical performance of the Mod-Pol-AR-AMF has been carried on in [16], in terms of false alarm probability $P_{f a}$ as well as detection probability $P_{d}$, for both a Swerling 0 and a Swerling I target model [24].

Specifically the performance of the detector have been derived in the case of perfectly known disturbance characteristics and the corresponding expressions are detailed in equations (30), (31) and (36) of the Appendix.

In addition, a practical $P_{f a}$ expression has been obtained to select a proper detection threshold $\eta$ that accounts for the fluctuations in the estimation of matrix $\widehat{\mathbf{D}}_{w}$ due to finite $P$

$$
P_{f a}=\frac{(1-\kappa)^{P-L+1}}{\Gamma(P-L+1)} \sum_{l=0}^{L-1} \frac{\Gamma(P-l)}{\Gamma(L-l)} \kappa^{L-l+1}
$$

where $\Gamma(\cdot)$ is the Gamma function and $\eta=P \frac{\kappa}{(1-\kappa)}$. Note that eq. (16) is obtained assuming that only the first adaptive cancellation stage meets the asymptotic conditions. In other words, in the development of the theoretical performance, we assumed that $K M$ is sufficiently high for matrices $\widehat{\mathbf{A}}$ and $\widehat{\mathbf{R}}$ to be asymptotic, though possibly mismatched, estimates of the AR parameters.

The direct implementation of the Mod-Pol-AR-AMF to the PCL case reveals three challenging aspects that need further investigations.

(i) First, the computational complexity of the detector must be carefully addressed. Note that, in passive radar applications, long CPIs (in the order of seconds) are typically used to attain desired levels of SNR. In fact, the number $M$ of samples included in the CPI is at least in the order of hundreds of thousands and this sets quite demanding computational requirements. Moreover, as shown by the explicit dependence on the CUT in (7)(8)-(10) and (14) the operations included therein must be repeated for each bistatic range and Doppler bin in the map where the presence of target is sought. 
Consequently, the considerable computational complexity is expected to prevent the direct implementation of the Mod-PolAR-AMF in (14) and suitable strategies should be investigated with the aim of obtaining an efficient implementation of the proposed detection scheme. The subsequent Section III will be devoted to the derivation of a cost-effective approximate version of the Mod-Pol-AR-AMF that simultaneously ensures negligible loss.

(ii) Moreover, the strategy adopted to adaptively adjust the filter weights must be considered. In a pulsed radar application, the considered detection scheme is applied after the range compression stage, thus allowing the extraction of a set of training data from $K$ adjacent range cells. Clearly, this is not possible in a PCL application, as we operate in the fasttime domain. Therefore, one possible solution could be to extract the secondary data from signal fragments adjoining the employed CPI and not used for detection purposes. However, the i.i.d. condition must be carefully verified especially in the presence of disturbance contributions whose spectral characteristics slowly change with time. In Section IV we will carry out a thorough validation of different possible secondary data selection and adaptivity strategies.

(iii) Finally, both the computational complexity and the adaptivity strategy will depend on the employed number of taps $Q$ or, equivalently, on the adopted AR model order $Q-1$. It is therefore clear that the purpose of a proper selection of this parameter is twofold: on the one hand we aim at approximating the spectral characteristics of the input disturbance and on the other hand we aim at limiting the adaptivity loss and computational burden. Section IV will also deal with this aspect, aiming at identifying useful guidelines for the selection of the relevant parameters.

\section{COST-EFFECTIVE IMPLEMENTATION OF THE MOD-POL-AR-AMF}

The direct implementation of the Mod-Pol-AR-AMF requires a very high computational complexity that might prevent it from being used in practical applications. To illustrate this point, we have decomposed the entire computational complexity into three main components:

1. AR parameters estimation: The cost required for the adaptive estimation of the AR parameters and evaluation of matrix $\widehat{\mathbf{P}}$. In detail, this contribution includes the ML estimates of $\widehat{\mathbf{A}}$ and $\widehat{\mathbf{R}}$ based on matrix $\widehat{\mathbf{Q}}$ according to equations (11)-(13) and the subsequent evaluation of the $L Q \times L Q$ matrix $\widehat{\mathbf{P}}=\widehat{\mathbf{H}}^{H} \widehat{\mathbf{R}}^{-1} \widehat{\mathbf{H}}$, with $\widehat{\mathbf{H}}=\left[\begin{array}{ll}-\widehat{\mathbf{A}}^{H} & \mathbf{I}_{L}\end{array}\right]$.

2. Pol-time filtering: The cost required for the application of the polarimetric and temporal filter to the data (see (15)). This includes the evaluation of the temporally whitened sequence of $L$-dimensional vectors $\mathbf{y}_{0}\left(m, \tau, f_{D}\right)=\boldsymbol{\Sigma}^{H}\left(m, \tau, f_{D}\right) \widehat{\mathbf{P}} \tilde{\mathbf{x}}_{0}(m)$, followed by the summation across consecutive samples to achieve the coherent integration of target echoes in time

$$
\text { domain, say } \mathbf{w}_{0}\left(\tau, f_{D}\right)=\sum_{m=0}^{M-Q} \mathbf{y}_{0}\left(m, \tau, f_{D}\right) \text {. }
$$

3. Polarimetric whitening and test: The cost required for the final polarimetric whitening stage based on matrix $\widehat{\mathbf{D}}_{w}$, followed by the detection test in (14).

The computational load expected for the direct implementation of the Mod-Pol-AR-AMF is reported in the first column of Table I. Specifically, we report the order of magnitude of the number of floating-point operations (FLOPs) needed for each of the three components above, expressed as a function of the relevant matrices size. We assume that a complex addition requires 2 FLOPs and a complex multiplication requires 6 FLOPs.

It is easy to verify that the main contribution to the cost is represented by the second component, i.e. the pol-time filtering stage. Basically, this cost increases both with the employed CPI length, namely the number of samples $M$, and with the extent of the range and Doppler region of interest, along with the number $L$ of polarimetric channels and the number $Q$ of taps. In fact, the computations included in point 2 above must be repeated at each delay and Doppler bin where the target echo is looked for. Notice that the total number of considered delay and Doppler bins $\left(N_{\tau}\right.$ and $N_{f}$, respectively) is typically in the order of $10^{4}-10^{5}$ when long range surveillance applications are considered.

Consequently, we look for an efficient implementation of the proposed detection algorithm, based on reasonable approximations. The sought strategy aims at reducing the computational burden while guaranteeing negligible or limited loss. To this purpose, we first observe that the dependence of the pol-time filter on the Doppler $f_{D}$ is only due to the matrix $\boldsymbol{\Sigma}\left(m, \tau, f_{D}\right)$ and, in turn, to the vector $\tilde{\mathbf{t}}\left(m, \tau, f_{D}\right)$ used to build that matrix (see (10)):

$$
\tilde{\mathbf{t}}\left(m, \tau, f_{D}\right)=\left[\begin{array}{c}
r\left(t_{0}+\frac{m}{f_{s}}-\tau\right) \\
\vdots \\
r\left(t_{0}+\frac{m+Q-1}{f_{S}}-\tau\right)
\end{array}\right] \odot\left[\begin{array}{c}
e^{j 2 \pi m \frac{f_{D}}{f_{s}}} \\
\vdots \\
e^{j 2 \pi(m+Q-1) \frac{f_{D}}{f_{s}}}
\end{array}\right]
$$

Assuming that the sub-CPI is short, i.e. for limited values of $Q$, this vector can be approximated as follows:

$$
\begin{gathered}
\tilde{\mathbf{t}}\left(m, \tau, f_{D}\right) \cong\left[\begin{array}{c}
r\left(t_{0}+\frac{m}{f_{s}}-\tau\right) \\
\vdots \\
r\left(t_{0}+\frac{m+Q-1}{f_{s}}-\tau\right)
\end{array}\right] e^{j 2 \pi m \frac{f_{D}}{f_{s}}} \\
=\tilde{\mathbf{t}}_{0}(m, \tau) e^{j 2 \pi m \frac{f_{D}}{f_{s}}}
\end{gathered}
$$

where we have defined the vector $\tilde{\mathbf{t}}_{0}(m, \tau)=\tilde{\mathbf{t}}(m, \tau, 0)$ and we have neglected the Doppler induced phase variation within the $Q$-dimensional sub-CPI. Consequently, by defining $\boldsymbol{\Sigma}_{0}(m, \tau)=\tilde{\mathbf{t}}_{0}(m, \tau) \otimes \mathbf{I}_{L}$, matrix $\boldsymbol{\Sigma}\left(m, \tau, f_{D}\right)$ becomes 
TABLE I

COMPUTATIONAL COMPLEXITY OF THE MOD-POL-AR-AMF (NUMBER OF FLOPS)

\begin{tabular}{ccc}
\hline & Direct Implementation & Cost-Effective Implementation \\
\hline $\begin{array}{c}\text { AR parameters } \\
\text { estimation }\end{array}$ & $O\left[4 K(M-Q+1)(L Q)^{2}\right]$ & $O\left[4 K(M-Q+1)(L Q)^{2}\right]$ \\
Pol-time filtering & $O\left[8 N_{\tau} N_{f}(M-Q+1) L^{2}(Q-1)\right]$ & $O\left[8 N_{\tau} L(M-Q+1)\left(\log _{2}(M-Q+1)+L Q\right)\right]$ \\
$\begin{array}{c}\text { Polarimetric whitening } \\
\text { and test }\end{array}$ & $O\left[N_{\tau} N_{f} P L(4 L+5)\right]$ & $O\left[N_{\tau} N_{f} P L(4 L+5)\right]$ \\
\hline
\end{tabular}

$$
\boldsymbol{\Sigma}\left(m, \tau, f_{D}\right) \cong \boldsymbol{\Sigma}_{0}(m, \tau) e^{j 2 \pi m \frac{f_{D}}{f_{s}}}
$$

and the pol-time filter output can be simplified as

$$
\mathbf{w}_{0}\left(\tau, f_{D}\right)=\sum_{m=0}^{M-Q} \boldsymbol{\Sigma}_{0}^{H}(m, \tau) \widehat{\mathbf{P}} \tilde{\mathbf{x}}_{0}(m) e^{j 2 \pi m \frac{f_{D}}{f_{s}}}
$$

where the computationally intense evaluation of the whitened sequence $\mathbf{y}_{0}(m, \tau)=\boldsymbol{\Sigma}_{0}^{H}(m, \tau) \widehat{\mathbf{P}} \widetilde{\mathbf{x}}_{0}(m)$, has not to be repeated at each Doppler bin. Instead, the Doppler dependent phase term can be taken into account at the subsequent coherent integration stage needed to obtain $\mathbf{w}_{0}\left(\tau, f_{D}\right)$. Assuming that the search grid in the Doppler dimension is uniformly spaced and not oversampled, for a given delay bin $\tau$, eq. (20) corresponds to the evaluation of $L$ Discrete Fourier Transforms (DFTs) of filtered sequences of $M-Q+1$ samples each. This could be easily implemented by resorting to a Fast Fourier Transform (FFT). By repeating the computations above across an appropriate grid of delays, $L$ range/Doppler maps are obtained, and the final adaptive detector is applied for each CUT. The resulting processing scheme is scketched in Fig.2.

Before evaluating the computational load saving, we first investigate the loss yield by the approximation above. In this regard, note that, if the target echo is such that

$$
\tilde{\mathbf{t}}\left(m, \tau, f_{D}\right)=\tilde{\mathbf{t}}\left(0, \tau, f_{D}\right) e^{j 2 \pi m \frac{f_{D}}{f_{S}}}
$$

the approximation in (18), which would imply that $\tilde{\mathbf{t}}\left(0, \tau, f_{D}\right) \cong \tilde{\mathbf{t}}_{0}(0, \tau)$, is irrelevant. In other words, under the condition in (21), neglecting the Doppler induced phase variation within the sub-CPI, does not modify the asymptotic detection performance of the simplified detector with respect to the detector in (14). See Appendix for demonstration.

This is, for instance, the case of a polarimetric active pulsed radar system where vector $\mathbf{t}$ represents the $M$ dimensional temporal steering vector and no tapering window is applied, namely if $\mathbf{t}$ encodes the target echo phase shifts across consecutive pulses.

On the other hand, when eq. (21) is not valid, e.g. in the case of a PCL system where $\mathbf{t}$ is a fragment of the transmitted signal, a loss might result from using the cost-effective implementation of the detector. However, we show in the following that, for the parameter values typical of PCL applications, the resulting loss is negligible.

To illustrate this point, we compare in Fig.3(a) the asymptotic performance obtained with the direct implementation of the Mod-Pol-AR-AMF and the costeffective implementation of the same detector for a simulated scenario. To this end, we consider the case of $L=2(\mathrm{H}, \mathrm{V})$ and a CPI of $M=10^{3}$ samples. The signal of opportunity is generated as an FM radio transmission [25] containing music content. We model the disturbance as a $L-$ channel $\operatorname{AR}(Q-1)$ process, with $Q=10$, a polarimetric correlation coefficient equal to 0.9 and equal spectral characteristics at the $\mathrm{H}$ and $\mathrm{V}$ channels. Two different target models are considered, namely Swerling 0 and Swerling I. According to a Swerling 0 target model [24], the target complex amplitudes are assumed deterministic.

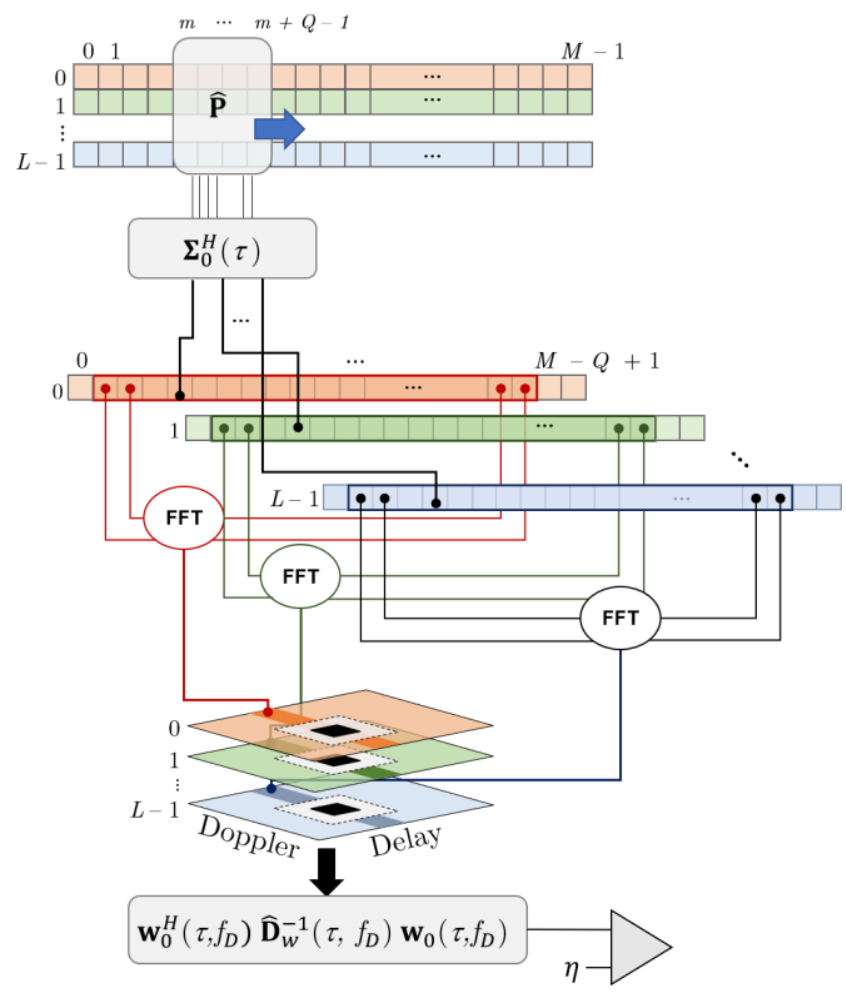

Fig.2 Sketch of the cost-effective implementation of Mod-Pol-AR-AMF. 


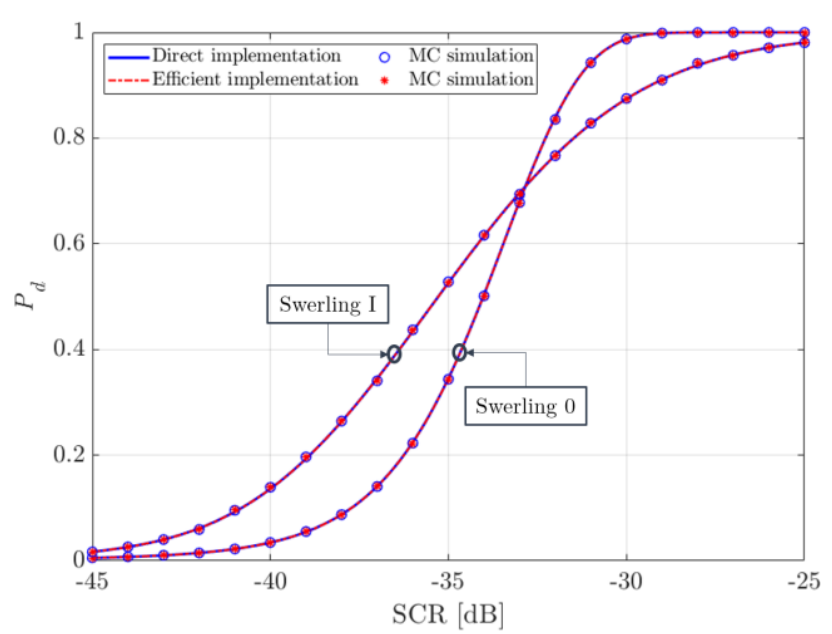

(a)

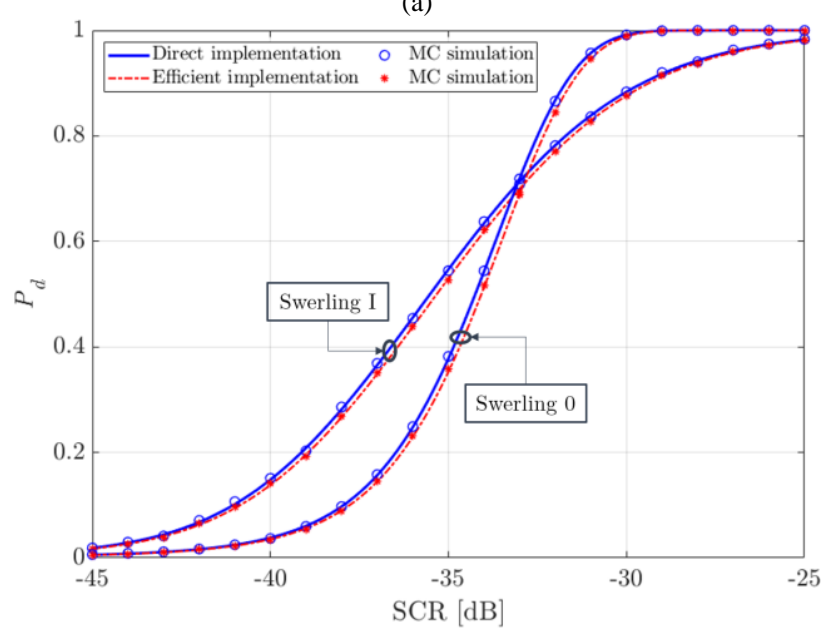

(b)

Fig.3 $P_{d}$ vs SCR with $P_{f a}=10^{-3}$ for an AR(9) process with target Doppler frequency equal to (a) $f_{D}=10^{-3} f_{S}$ and (b) $f_{D}=10^{-2} f_{S}$.

In the considered case study, they are set as $\boldsymbol{\alpha}=$ $a_{t}\left[1 \quad \mathrm{e}^{j \Delta \phi_{H / V}}\right]^{T}$, with $\Delta \phi_{H / V}=\pi / 4$. For the Swerling I target model [24], the target complex amplitudes vector $\boldsymbol{\alpha}$ is generated as a zero-mean Gaussian random vector, with the following covariance matrix

$$
\mathbf{M}_{t}=E\left\{\boldsymbol{\alpha} \boldsymbol{\alpha}^{H}\right\}=\sigma_{t}^{2}\left(\begin{array}{ll}
1 & 0 \\
0 & 1
\end{array}\right)
$$

We report in Fig.3(a) the asymptotic $P_{d}$ versus the input signal-to-clutter ratio (SCR) for a target with Doppler frequency equal to $f_{D}=10^{-3} f_{S}$. The SCR at the first polarimetric channel is considered as a reference and it is obtained as $\mathrm{SCR}=\left|a_{t}\right|^{2} / \sigma_{d}^{2}$ and as $\mathrm{SCR}=\sigma_{t}^{2} / \sigma_{d}^{2}$ for the non-fluctuating and fluctuating target model, respectively, being $\sigma_{d}^{2}$ the disturbance power, deliberately set to $\sigma_{d}^{2}=1$ at both polarimetric channels. Also the results of a Monte Carlo (MC) simulation are reported to prove the validity of the theoretical $P_{d}$ expressions.
By observing Fig.3(a), we note that the asymptotic $P_{d}$ curves obtained with the direct implementation of the detector (blue lines and markers) are substantially identical to those obtained with the cost-effective solution (red lines and markers) for any of the two considered target models.

The loss is expected to increase with the Doppler frequency of the considered target echo as the approximation in (18) becomes weaker. This is shown in Fig.3(b) where we extended the theoretical performance analysis to the case of a target with Doppler frequency equal to $f_{D}=10^{-2} f_{S}$. As it is apparent, the loss is still negligible and limited to $0.2 \mathrm{~dB}$. Besides we observe that, using typical parameters for a FM radio based PCL system, e.g. the ones reported in Table II, the employed target Doppler frequency value would correspond to a bistatic velocity of approx. $6000 \mathrm{~m} / \mathrm{s}$ which is well outside the range of practical values for typical surveillance applications. Consequently, based on the considerations on Fig.3, we can infer that the cost-effective implementation of the Mod-Pol-AR-AMF yields negligible loss for the typical operative scenarios.

Therefore, it is worth investigating the obtained computational complexity reduction. To this aim, we use the same decomposition presented at the beginning of this Section and we report the order of magnitude of FLOPs required for each component of the cost in the second column of Table I.

As expected, the cost required for the second component significantly reduces when the proposed approximation is adopted with respect to the direct implementation of the ModPol-AR-AMF. In contrast, we recall that the cost required for the first and the third components are not affected by the proposed simplification. Specifically, the cost for the AR parameters estimation is mainly represented by the number of complex operations required for the estimation of matrix $\widehat{\mathbf{Q}}$ according to (13) while the cost required for the polarimetric whitening and detection test is mainly given by the estimation and inversion of the $L \times L$ matrix $\widehat{\mathbf{D}}_{w}$, based on $P$ training data, to be repeated $N_{\tau} N_{f}$ times.

To further investigate how the computational complexity decreases with the efficient implementation, a dedicated numerical analysis is required for practical cases.

To this purpose, we refer to the case of a PCL system for aerial surveillance exploiting FM radio transmissions. The employed parameters are reported in Table II.

TABLE II

SELECTED PARAMETERS FOR THE COMPUTATIONAL COMPLEXITY EVALUATION

\begin{tabular}{cc}
\hline Parameter & Value \\
\hline Carrier frequency $f_{C}[\mathrm{~Hz}]$ & $94.5 \cdot 10^{6}$ \\
Sampling frequency $f_{S}[\mathrm{~Hz}]$ & $200 \cdot 10^{3}$ \\
Polarimetric channels $L$ & 2 \\
Max. relative bistatic range $[\mathrm{km}]$ & 200 \\
Max. bistatic velocity $[\mathrm{m} / \mathrm{s}]$ & 500 \\
Temporal training data $K$ & 1 \\
Secondary data $P$ & 32 \\
\hline
\end{tabular}




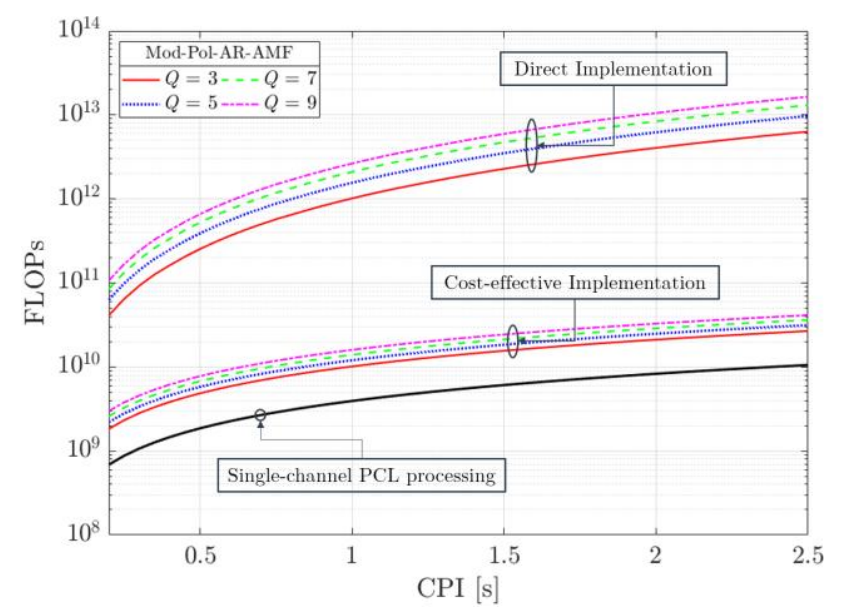

Fig.4 Computational complexity versus CPI for the direct implementation and the cost-effective version of the Mod-Pol-AR-AMF. The employed parameters are reported in Table II.

Note that we assume the availability of $K=1$ secondary data vector, which includes $M$ samples of the signals simultaneously collected at the $L=2$ polarimetric channels, and it is used as training data for the AR parameters estimation. Correspondingly, we report in Fig.4 the total cost required for the Mod-Pol-AR-AMF based on the direct and cost-effective implementations of the detector versus the CPI length for different values of $Q$. We also report in bold black line the number of FLOPs required for a conventional singlechannel PCL processing scheme after the first disturbance cancellation stage for comparison. Specifically this encompasses (i) the evaluation of a bistatic range - velocity map and (ii) a cell average (CA-CFAR) detection scheme with $P$ training data used to adaptively scale the threshold. In detail, the evaluation of the range-velocity map is assumed to be carried out using an efficient algorithm described in [4] and referred to as Direct-FFT which requires $N_{\tau}\left(8 M \log _{2}(M)+\right.$ $6 M$ ) FLOPs.

As expected, Fig.4 shows that:

- the number of FLOPs required for the direct implementation of the Mod-Pol-AR-AMF is much higher than for the singlechannel processing and their separation grows with $Q$. The increase is approximately of three orders of magnitude in the considered case study and might be higher if we include in the overall computational complexity the cost of the temporal disturbance cancellation stage [22], preliminarily separately applied to each of the surveillance signals regardless of the employed detector.

- The computational complexity required for the costeffective implementation of the Mod-Pol-AR-AMF is substantially lower. For instance, the reduction is larger than two orders of magnitude for typical values of the CPI length, namely $1-2 \mathrm{~s}$.

The cost-effective implementation of the Mod-Pol-ARAMF will be used in the following to carry out an extensive performance analysis against real data aiming at validating the expected improvement with respect to both single-pol PCL system and alternative polarimetric approaches. Incidentally we observe that the same analysis would have been unfeasible using the direct implementation of the algorithm.

\section{TUNing OF THE RELEVANT PARAMETES}

In this Section, we carry out an extensive analysis of the performance of the Mod-Pol-AR-AMF on real data, enabled by the cost-effective implementation proposed in Section III. The objective of this analysis is to provide further insights into the proposed detector by investigating the remaining challenging aspects of its application to the passive radar case, as listed in Section II.B.

\section{A. Experimental data and preliminary results}

To this aim, we use the same experimental data employed in [8] and collected during an experimental campaign carried out near Fiumicino Airport, in Italy, exploiting the FM radio transmitter of Monte Cavo as IO, located approx. $35 \mathrm{~km}$ away from the PCL receiver, see Fig.5(a). Fig.5(b) shows the two employed dual-polarized log periodic antennas, each equipped with two independent outputs, one vertical (V) and one horizontal $(\mathrm{H})$ polarized.

One of them was used as reference antenna and steered toward the $\mathrm{IO}$ to collect the $\mathrm{V}$ and $\mathrm{H}$ polarized versions of the transmitted signal. The other was adopted as surveillance antenna and was pointed toward the area to be monitored,

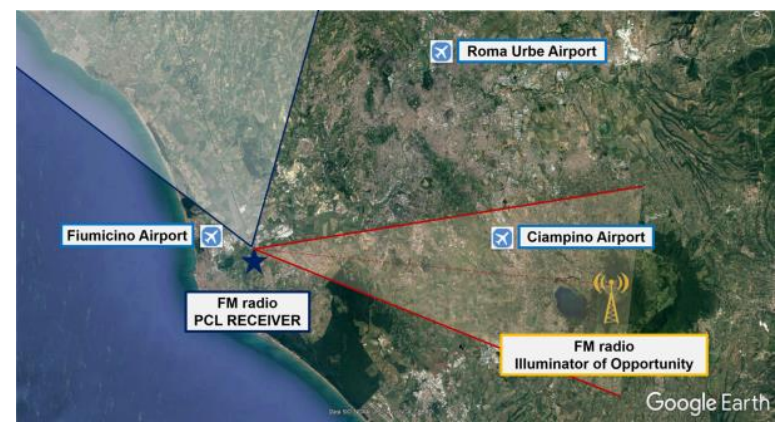

(a)

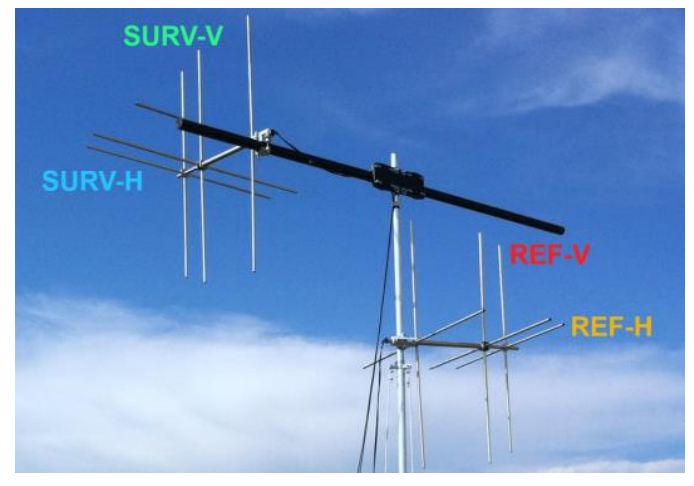

(b)

Fig.5 Acquisition campaign:

(a) geometry (b) employed dual-polarized antennas. 
simultaneously collecting target echoes at the $\mathrm{V}$ and $\mathrm{H}$ channel. The employed data set consists of 2060 data files, each containing a $1.1 \mathrm{~s}$ registration of the signals simultaneously collected by the different antennas. A total acquisition of 80 minutes was obtained, using a multi-channel PCL prototype, based on a direct RF sampling approach and exploiting the ICS-554 PMC module (GE Fanuc Embedded Systems). This module consists of four 14-bit ADCs sampling synchronously the properly amplified and filtered analogue signals from up to four input channels. Simultaneous down conversion of up to 16 arbitrary signal bands (e.g. 16 FM radio channels) is provided by four Graychip GC4016 quad digital downconverters (DDC). The described setup allows collecting data from up to four different FM radio channels from each receiving antenna. In this work, we consider the FM channel at $94.5 \mathrm{MHz}$, transmitted with $\mathrm{V}$ polarization. The air-truth for the same air space has been provided by the SBS-1 real time virtual radar.All the available data files first underwent the Pol-ECA temporal disturbance cancellation stage described in [8] with a filter length 140 taps.

Afterwards, different processing schemes are applied and compared, using a CPI of $1 \mathrm{~s}$.

To preliminarily investigate the benefits of the joint exploitation of different polarimetric channels, we report in Fig.6(a-c) the raw detection results obtained for 50 consecutive data files over the same bistatic range-velocity plane with nominal $P_{f a}=10^{-5}$. The gray lines represent the available air-truth while the blue dots denote the raw PCL detections.

In detail, Fig.6(a-b) are obtained when the conventional single-channel PCL processing is separately applied to each of the surveillance signals. By comparing Fig.6 (a) and (b), we notice that the $\mathrm{V}$ polarization outperforms the $\mathrm{H}$ polarization for the considered dataset. However, even with the best performing channel, we observe that only a few isolated plots are obtained for some of the target tracks. Fig.6(c) shows the result obtained when using the proposed Mod-Pol-AR-AMF, with $L=2$ and $Q=3$. We used a signal fragment adjoining the signal samples included in the CPI, to estimate the AR parameters. Specifically, a fragment composed by $J+Q-1$ samples, or equivalently by $J$ subsequent overlapped subCPIs, is employed, with $J=200$. Fig. 6 clearly shows that when the polarimetric information is exploited according to the proposed detection scheme, the target detection capability is remarkably improved with respect to the use of single polarimetric channels. In fact, longer and much denser sequences of detections are obtained in Fig.6(c).

As mentioned, the promising results of Fig.6 have been obtained over a set of few consecutive data files with a given selection of the processing parameters. In the following, the results of extended performance analyses are reported based on the entire dataset of 2060 files and the available air-truth. Specifically, the empirical receiver operating characteristic (ROC) curves have been evaluated to demonstrate the relative frequency of target detections against a grid of false alarm rate values. At each data file, and for each considered false alarm rate value, a correct detection was declared when a peak in the range-Doppler plane exceeded the selected threshold at the radar coordinates predicted by the available air-truth plus a small tolerance.

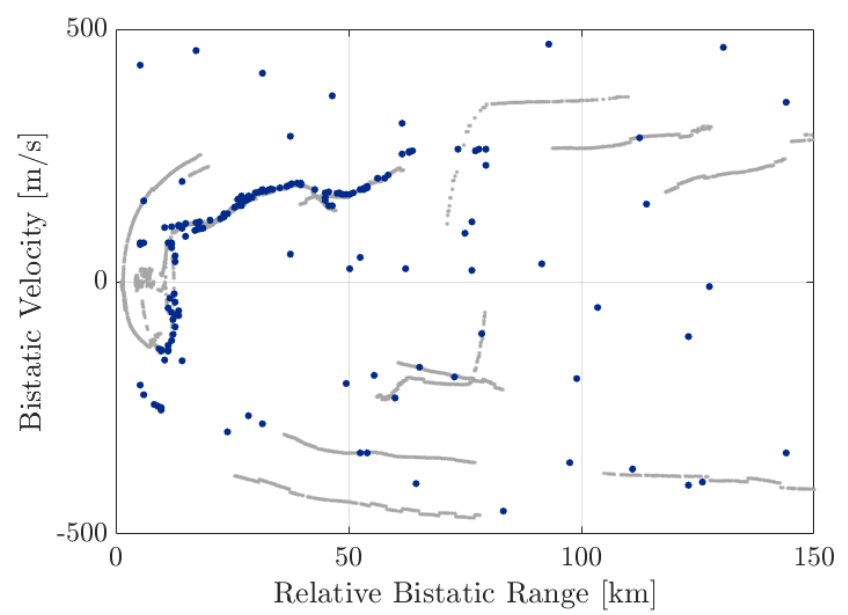

(a)

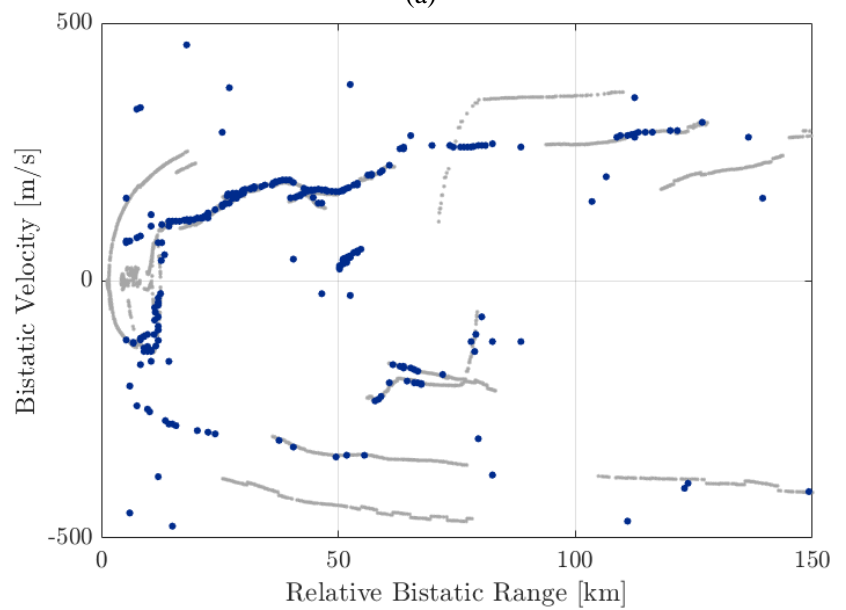

(b)

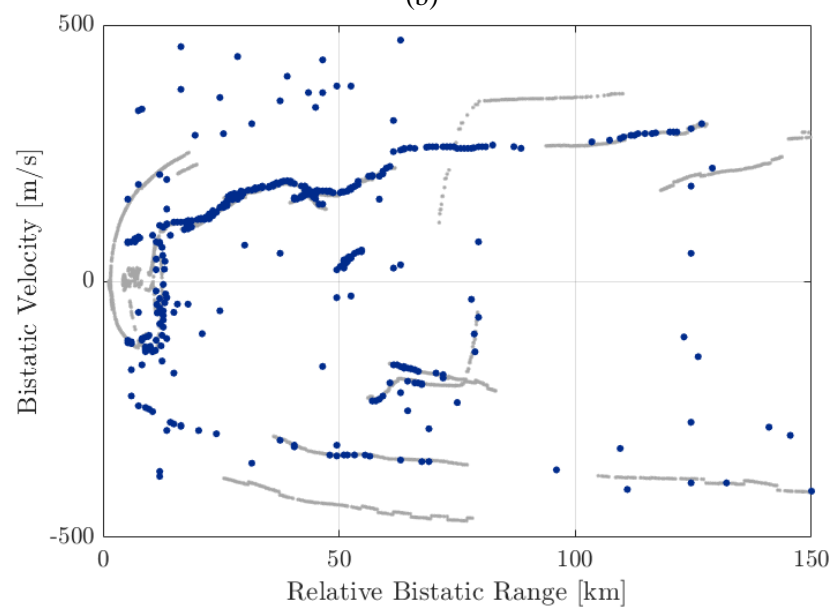

(c)

Fig.6 Detection results over 50 consecutive data files with $P_{f a}=10^{-5}$, using (a) Single-pol H (b) Single-pol V

(c) Mod-Pol-AR-AMF with $Q=3$ and $J=200$ (S1). 
Then, the detection frequency is obtained by dividing the number of correct detections by the maximum number of target occurrences, namely 9632 . Note that the detection analysis is limited to targets laying in the range-band [0-100] $\mathrm{km}$ and included within an angular sector of $90^{\circ}$ around the surveillance antenna pointing direction. All other peaks exceeding the threshold are considered false alarms. The false alarm density is measured in the range band [150-200] km where the detection probability is very low so that it is unlikely to include and label as false alarms detections corresponding to targets not equipped with a transponder and therefore not identified by the available air-truth, see e.g. the track at approx. $250 \mathrm{~m} / \mathrm{s}$ between 60 and $90 \mathrm{~km}$ in Fig.6(c).

The methodology above is exploited to investigate the impact of the processing parameters on the average performance of the Mod-Pol-AR-AMF. First, we investigate alternative strategies to estimate the AR parameters and thus to adaptively adjust the pol-time filter weights (sub-Section IV.B). Then, we study the effect of diverse choices of the relevant parameters (sub-Section IV.C).

\section{B. Adaptivity Strategies}

In Section II, we assumed that the adaptive AR parameters estimation is obtained by relying on the availability of $K$ i.i.d. and target free secondary vectors $\mathbf{x}_{k}, k=1, \ldots, K$. However, we mentioned that this strategy must be revised to allow the application of the proposed detector to the passive radar case.

\section{1) Strategy \#1 (S1)}

As previously introduced, among the possible adaptivity strategies, a suitable solution is to extract the secondary data from a signal fragment adjoining the employed CPI (primary data) and not used for the detection. Specifically, we assume $J+Q-1$ data samples are available, including $J$ consecutive overlapped sub-CPIs of dimension $Q$. Accordingly, eq. (13) is modified as

$$
\widehat{\mathbf{Q}}=\sum_{j=M}^{M+J-1} \tilde{\mathbf{x}}_{0}(j) \tilde{\mathbf{x}}_{0}^{H}(j)
$$

Once the covariance matrix $\widehat{\mathbf{Q}}$ is estimated according to (23), the AR parameters are evaluated as in (11)-(12) and the results are used for the evaluation of matrix $\widehat{\mathbf{P}}$. For instance, this is the strategy adopted in Fig.6(c). S1 certainly guarantees the statistical independence of the primary and secondary data. However, since very long CPIs are typically used in passive radar applications, this strategy might result in a training data set that is no longer representative of the disturbance affecting the primary data. In fact, the temporal separation between the primary and secondary data might jeopardize the hypothesis of identical distribution since the disturbance process cannot be assumed stationary over long periods.

As an alternative, portions of the CPI might be used to adaptively adjust the AR parameters to be employed in the filter evaluation. In particular, two possible solutions are considered in this paper:

\section{2) Strategy \#2 (S2)}

The estimation of the covariance matrix $\widehat{\mathbf{Q}}$ is carried out using the whole primary data, averaging the estimation over the entire CPI:

$$
\widehat{\mathbf{Q}}=\sum_{m=0}^{M-Q} \tilde{\mathbf{x}}_{0}(m) \tilde{\mathbf{x}}_{0}^{H}(m)
$$

Then, using the result of (24), matrices $\widehat{\mathbf{A}}$ and $\widehat{\mathbf{R}}$ are derived as in (11)-(12) and plugged into the expression of matrix $\widehat{\mathbf{P}}$. $\mathrm{S} 2$ is based on the simplifying assumptions that, due to the high number of samples in the CPI, the target contributions are negligible with respect to the competing disturbance and an asymptotic estimate of the AR parameters is achieved.

\section{3) Strategy \#3 (S3)}

The simplifying assumptions of S2 might hold even when reasonably reducing the size $J$ of the training set with respect to the whole CPI length $M$, especially when the size of the matrix to be estimated is kept small, namely for limited $Q L$ product. However, using smaller portions of the CPI allows to update the estimate of matrix $\mathbf{Q}$ across the integration time thus increasing the robustness against disturbance contributions whose spectral characteristics slowly change with time. Therefore, in S3, eq. (13) is modified as:

$$
\widehat{\mathbf{Q}}_{n}=\sum_{\substack{m=n J \\(n=0, \ldots, N-1)}}^{(n+1) J-1} \tilde{\mathbf{x}}_{0}(m) \tilde{\mathbf{x}}_{0}^{H}(m)
$$

where we assumed that the estimation of matrix $\mathbf{Q}$ is repeated $N=\lceil(M-Q+1) / J\rceil$ times. At the $n$-th block of $J$ sub-CPIs, the AR parameters $\widehat{\mathbf{A}}_{n}$ and $\widehat{\mathbf{R}}_{n}$ are updated according to (11)(12) and used to evaluate $\widehat{\mathbf{P}}_{n}=\widehat{\mathbf{H}}_{n}^{H} \widehat{\mathbf{R}}_{n}^{-1} \widehat{\mathbf{H}}_{n}$, where $\widehat{\mathbf{H}}_{n}=$ $\left[\begin{array}{ll}-\widehat{\mathbf{A}}_{n}^{H} & \mathbf{I}_{L}\end{array}\right]$. Accordingly, eq.(20) is modified as

$$
\mathbf{w}_{0}\left(\tau, f_{D}\right)=\sum_{m=0}^{M-Q} \boldsymbol{\Sigma}_{0}^{H}(m, \tau) \widehat{\mathbf{P}}(m) \tilde{\mathbf{x}}_{0}(m) e^{j 2 \pi m \frac{f_{D}}{f_{s}}}
$$

where the filter weights update across subsequent sub-CPIs is made explicit and the matrix $\widehat{\mathbf{P}}(m)$ to be exploited at the $m$ th sub-CPI, selected as $\widehat{\mathbf{P}}(m)=\widehat{\mathbf{P}}_{[m / J]}$.

The three adaptivity strategies above are sketched in Fig.7 where we show the different approaches to the selection of the training set and have been validated and compared based on their application on real data. Alternative strategies that update the filter weights on partially overlapped portions of the CPI could be the subject for future investigation.

Note that, depending on the selected adaptivity strategy, the computational complexity required for the AR parameters estimation (see the first row of Table I) might slightly change More precisely, it is equal to $O\left[4 \mathrm{~J}(L Q)^{2}\right]$ for $\mathrm{S} 1, O[4(M-Q$ $\left.+1)(L Q)^{2}\right]$ for $\mathrm{S} 2$ and $O\left[4 N J(L Q)^{2}\right]$ for S3. Therefore, if 


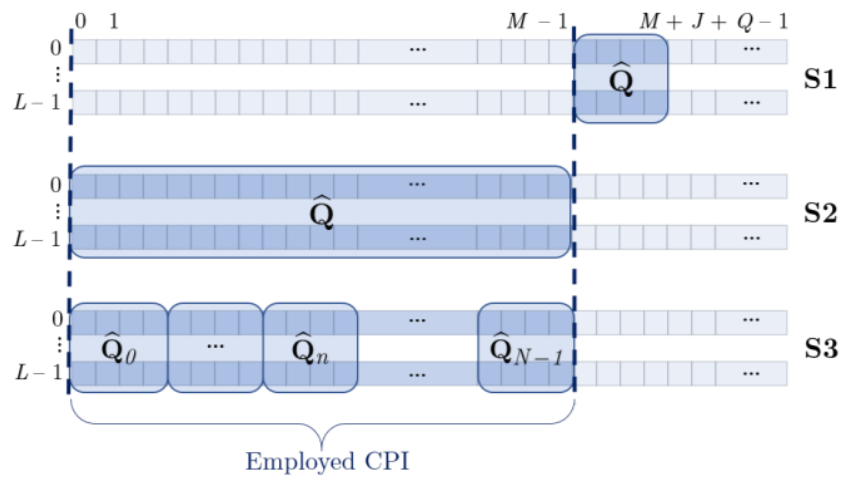

Fig.7 Sketch of the considered adaptivity strategies.

$J<<M-Q+1, \mathrm{~S} 1$ requires the lowest computational load while the cost is comparable for $\mathrm{S} 2$ and $\mathrm{S} 3$. However, as mentioned, the most demanding cost component is represented by the poltime filtering (see second row of Table I) therefore the, despite the slight potential variation, the overall complexity is comparable for the three cases.

Fig.8 reports the empirical ROC curves obtained for the Mod-Pol-AR-AMF with $Q=3$, exploiting S1, S2, or S3 against the available datasets. We set $J=200$ in S1 and S3. The results obtained with the single-pol operation at both the $\mathrm{H}$ and $\mathrm{V}$ channel are also shown for comparison. Observing Fig.8, first we can confirm what already demonstrated in [8],[10] and [17] and shown in Fig.6(a-b), namely that the target detection capability obtained using the conventional single-channel processing is different when using the $\mathrm{V}$ or the $\mathrm{H}$ channel. With reference to this dataset, the $\mathrm{V}$ polarization is the best performing single channel on average, however it was shown in [8],[10] that it is not possible to a priori establish the best performing channel.

It is also evident that the Mod-Pol-AR-AMF remarkably improves the detection capability with all considered adaptivity strategies, increasing the target detection rate up to $30 \%$ and $80 \%$ with respect to the $\mathrm{V}$ and $\mathrm{H}$ channels, respectively. Furthermore, among the considered strategies, namely comparing the magenta, blue, and red lines, it is clear that S3 yields the highest target detection rate. This result reveals the need to account for the non-stationary nature of the disturbance across the entire CPI. This is also confirmed by the fact that the worst performing adaptivity strategy is S1, namely the one that extracts the secondary data from a signal fragment temporally adjoining the considered CPI of $1 \mathrm{~s}$. Note that an increase of approx. $10 \%$ is obtained at $P_{f a}=10^{-4}$ with the $S 3$ with respect to $S 1$, which in turns results in +655 correct detections in the considered scenario.

\section{Setting of $Q$ and training data set}

S3 will be further investigated in this section in conjunction with the setting of the number $Q$ of taps of the pol-time filter. Specifically, different choices are considered

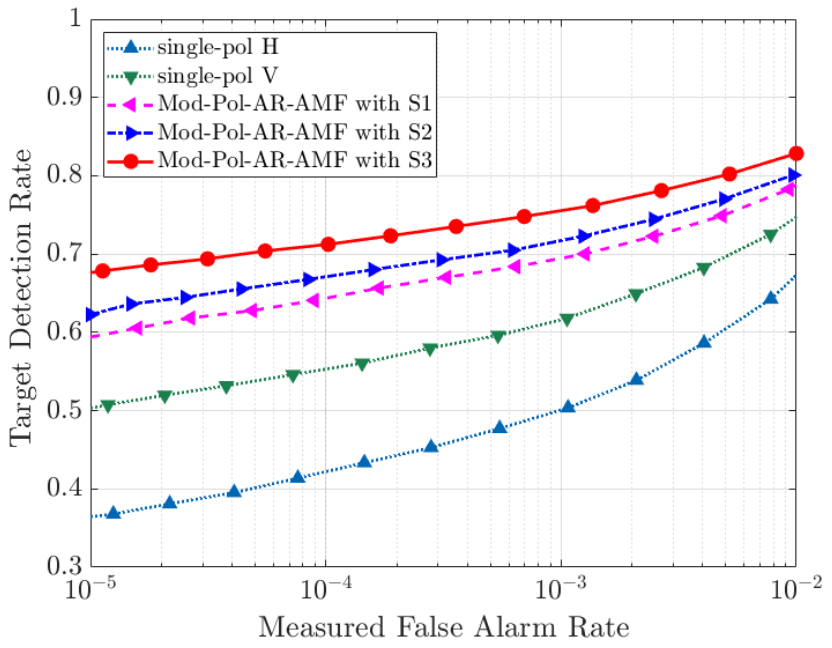

Fig.8 Empirical ROC curves with different detection schemes exploiting different adaptivity strategies for the Mod-Pol-AR-AMF.

for the selection of the training data size $J$. Note that when $J=$ $M-Q+1$, S3 coincides with S2.

For each combination of parameters, we repeated the extensive analysis yielding the empirical ROC curves. We collect the obtained results in Fig.9, that shows the target detection rate measured in the range band [0-100] $\mathrm{km}$ for a measured $P_{f a}$ equal to $10^{-3}$.

Specifically, each reported curve collects the results obtained with different $Q$ values for a given selection of the training data size $J$. To better appreciate the differences between the considered solutions, the $y$-axis has been limited between 0.65 and 0.8 .

Observing Fig. 9 the following considerations are in order: - An update of the AR parameters estimation along the CPI (S3) always allows an higher target detection performance with respect to using the entire CPI (S2). This confirms the comments on Fig.8 and the slowly varying characteristics of the disturbance.

- As the number $J$ of training data decreases and the number $Q$ of taps grows, the target detection capability suffers from incrisingly higher loss. The latter is due to both a higher adaptivity loss due to a limited training set and the assumption of negligible target echo that is likely to be no longer verified. In addition, we also recall that the sub-CPIs averaged to estimate the AR parameters are partially overlapped so that they do not provide independent training data and the percentage of overlap increases with $Q$. These effects are particularly evident when considering a relative small $J$, e.g. 50 or 100 . As $J$ increases, the same effects are expected to arise for higher $Q$.

- The target detection performance always improves moving from $Q=1$, namely when the employed filter only exploits the polarimetric diversity, to $Q \geq 2$. This confirms that the temporal correlation of the disturbance should be taken into account to effectively help in its rejection thus increasing the capability to discriminate targets. 


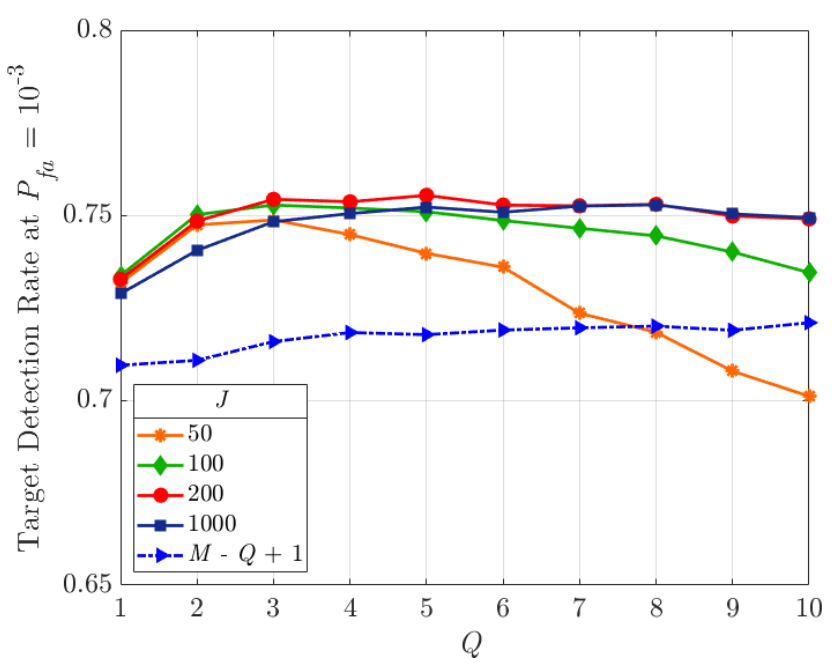

Fig.9 Target detection rate versus $Q$ with measured $P_{f a}=10^{-3}$ exploiting the adaptivity strategy $\mathrm{S} 3$ with different $J$.

- The solutions with $J=100,200$ and 1000 provide largely comparable performance in the interval between $Q=3$ and $Q=7$, with the maximum difference in terms of correct target detections being lower than 70 . We recall that this result is obtained by averaging the performance over the entire considered dataset, therefore specific improvements are not noticeable. Moreover, the local performance might be affected by a number of aspects that occur in pratical scenarios (e.g. low target altitudes, fluctuating target radar cross section). Therefore, whilst it cannot be deemed as an outright result, this shows the benefits of the proposed strategy when the relevant parameters are selected among an appropriate range. At this point, the choice of the employed parameters is also driven by further considerations, including the overall complexity. Therefore, we use the combination of $Q=3$ and the adaptivity strategy S3 with $J$ $=200$ in the following for the comparison with state-of-theart strategies that exploit polarimetric diversity.

\section{COMParison With STATE-OF-THE-ART Polarimetric DETECTION SCHEMES FOR PASSIVE RADAR}

In this Section, we compare the performance of the proposed detector with alternative existing solutions. The considered techniques, namely the polarimetric $\mathrm{NCI}$ and the polarimetric GLRT [8], are briefly summarized in the following and then encompassed in a thorough comparison that takes into account both the achievable detection performance and the required computational complexity.

Both the considered techniques operate in the delayDoppler, or equivalently, the bistatic range-velocity domain. Specifically, the $L$ polarimetric surveillance signals first undergo the Pol-ECA stage and then are separately used to evaluate $L$ range-velocity maps. These are finally exploited to implement two different detection strategies.

Under the simplifying assumption of statistically independent and identically distributed interference affecting different channels, the simplest approach consists in an NCI of the $L$ maps. To this purpose, the complex valued outputs obtained at a given delay-Doppler location are gathered in a $L$-dimensional vector $\chi\left(\tau, f_{D}\right)=\left[\psi_{0}\left(\tau, f_{D}\right) \ldots \psi_{L-1}\left(\tau, f_{D}\right)\right]^{T}$. The NCI stage sums up, after square law detector, the components of vector $\chi\left(\tau, f_{D}\right)$. Then, target detection is performed by resorting to a CA - CFAR scheme:

$$
\left\|\chi\left(\tau, f_{D}\right)\right\|^{2} \underset{H_{0}}{\stackrel{H_{1}}{\gtrless}} \eta_{\text {Pol-NCI}} \cdot \sum_{p \in I\left(\tau, f_{D}\right)}\left\|\chi\left(\tau_{p}, f_{D_{p}}\right)\right\|^{2}
$$

where $\chi\left(\tau, f_{D}\right)$ accounts for the range-Doppler CUT, $\eta_{P o l-N C I}$ is readily found by inverting the theoretical expression of the $P_{f a}$ in [8], and $I_{\left(\tau, f_{D}\right)}$ denotes a set of $P$ indices that identify range-Doppler bins surrounding the CUT from which the secondary cells are extracted, i.e. $\chi\left(\tau_{p}, f_{D_{p}}\right)(p \in$ $\left.I_{\left(\tau, f_{D}\right)},\left|I_{\left(\tau, f_{D}\right)}\right|=P\right)$. The detection scheme in (27) is referred to as Pol-NCI and it was shown in [5]-[8] that it allows a target echo enhancement. However, the weakness of the simplifying hypothesis of independent disturbance underlying this approach is revealed in a decreased capability of controlling the false alarm rate.

Based on these considerations, a polarimetric adaptive approach has been derived in [8] by resorting to a GLRT approach. The detection test is obtained as:

$$
\chi^{H}\left(\tau, f_{D}\right) \widehat{\mathbf{M}}^{-1}\left(\tau, f_{D}\right) \chi\left(\tau, f_{D}\right) \underset{H_{0}}{\stackrel{H_{1}}{\gtrless}} \eta_{P o l-G L R T}
$$

where $\widehat{\mathbf{M}}\left(\tau, f_{D}\right)=\frac{1}{P} \sum_{p \in I_{\left(\tau, f_{D}\right)}} \chi\left(\tau_{p}, f_{D_{p}}\right) \chi^{H}\left(\tau_{p}, f_{D_{p}}\right)$ is the sample covariance matrix, estimated via the $P$ training data $\chi\left(\tau_{p}, f_{D_{p}}\right), p \in I_{\left(\tau, f_{D}\right)},\left|I_{\left(\tau, f_{D}\right)}\right|=P$, and the threshold $\eta_{P o l-G L R T}$ is selected according to the $P_{f a}$ expression in (16). The detection scheme in (28) is referred to as Pol-GLRT [8] and it has been shown to effectively exploit the polarimetric information to improve the target discrimination capability, both using FM radio [8] and DVB-T signals [9][10]. Note that the detection test of the Pol-GLRT is similar to the one of the Mod-Pol-AR-AMF. In fact, both strategies encompass, as a final stage, the cascade of a polarimetric whitening, an NCI across the polarimetric dimension and a thresholding stage. However, according to the Pol-GLRT scheme, no previous pol-time filtering has been applied to the data before the range-velocity map evaluation.

Before comparing the performance of the proposed detector with the results of the Pol-GLRT and Pol-NCI, we study the computational complexity required by the mentioned strategies. The processing scheme of both the considered competing approaches requires (i) the evaluation of $L$ bistatic range - velocity maps and (ii) the application of the specific detection test. 


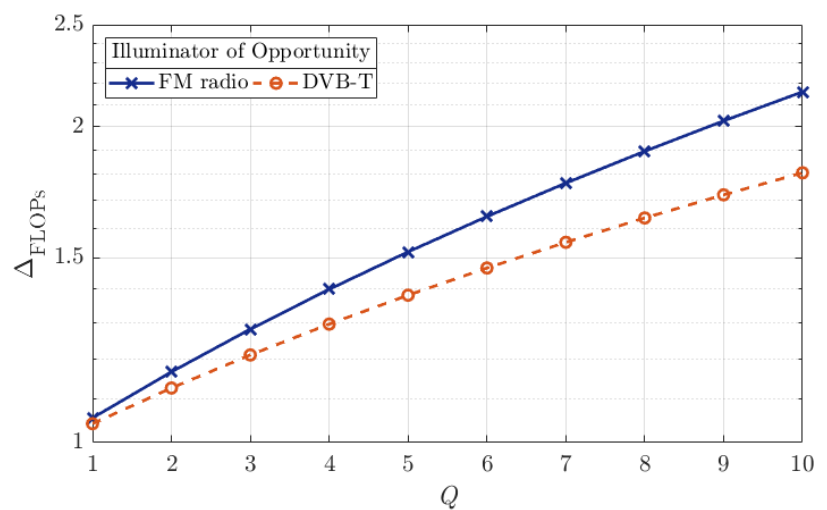

Fig.10 $\Delta_{\mathrm{FLOPS}}$ versus $Q$ exploiting FM radio and DVB-T signals.

As for the single-channel processing scheme in Section II, we assume that each map is evaluated using the efficient DirectFFT algorithm which requires $\left[N_{\tau}\left(8 M \log _{2}(M)+6 M\right)\right]$ FLOPs. Afterwards, with the Pol-NCI, a simple 2D autogate is applied to generate the adaptive threshold to be exploited for target detection. A slightly higher cost is expected with the Pol-GLRT since the corresponding detection scheme requires the same computational complexity of the 'polarimetric whitening and test' component detailed in Section III and reported in Table I. However, in both cases, the major contribution to the final computational complexity is represented by the evaluation of $L$ CAFs.

In Fig. 10 we study the surplus cost required by the ModPol-AR-AMF with respect to the Pol-GLRT. Specifically, the $\Delta_{\text {FLOPs }}$ is reported, defined as the ratio between the computational cost required by the Mod-Pol-AR-AMF and that needed to implement a Pol-GLRT, deducting from the overall complexity the cost required for the temporal disturbance cancellation stage, separately applied to the $L$

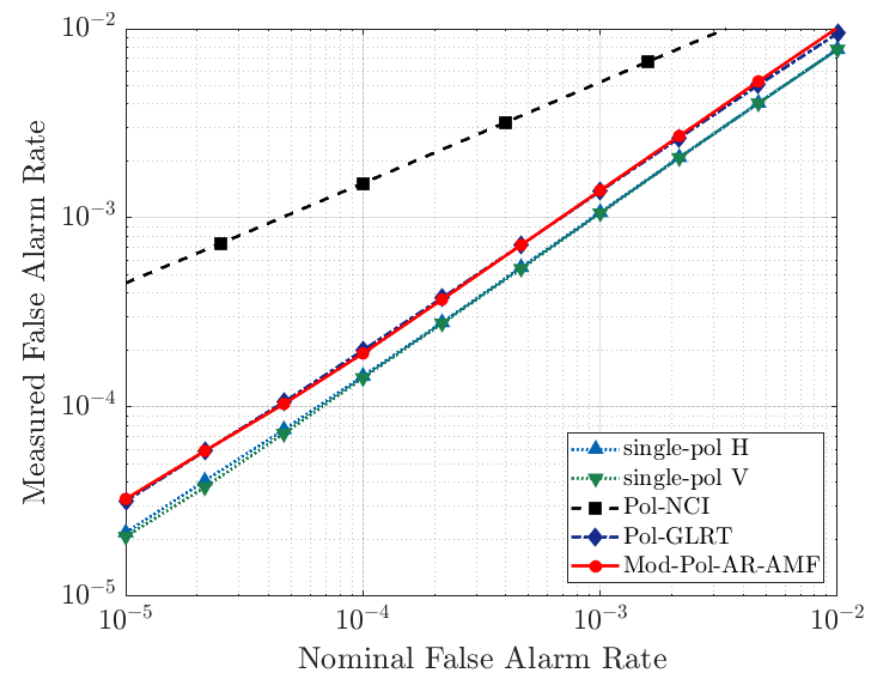

Fig.11 Measured versus nominal false alarm rate curves with different detection strategies for the bistatic range band [150-200] km. polarimetric channels regardless of the employed detector. The results are reported versus a grid of $Q$ values, using a CPI of 1s. The Mod-Pol-AR-AMF is assumed to operate with $J=$ 200 and the adaptivity strategy S3 whereas $P=32$ has been used for both approaches. The dark blue line represent the case of a PCL system exploiting FM radio transmissions, with the system parameters reported in Table II . This curve confirms that the Mod-Pol-AR-AMF requires a higher computational complexity with respect to the Pol-GLRT in the scenario considered in this paper. However, the complexity is less than doubled, i.e. $\Delta_{\text {FLOPs }}<2$, for the typical $Q$ values that have been shown to reasonably approximate the disturbance spectral characteristics. Note that surplus cost might further reduce if we take into account the burden of the preliminary temporal disturbance cancellation stage in evaluating of the overall complexity.

The case of a PCL system exploiting DVB-T signals is also reported for comparison. In the latter case, we assume that the signal is transmitted at carrier frequency $f_{C}=666 \mathrm{MHz}$ and received with sampling frequency $f_{S}=6817 / 896 \mathrm{MHz}$. The same extent of the range-velocity map is considered as in Table II. Incidentally, we notice that in this case the computational load reduction offered by the cost-effective implementation of the detector is even higher. In fact, it is equal to approx. three orders of magnitude and this allows to restore a computational complexity that is comparable to that required by the Pol-GLRT in the same scenario. A dedicated analysis would be required to investigate the benefits of the proposed detector when exploiting DVB-T signals of opportunity.

In this paper we exploit the available dataset to carry out an extensive comparison for the case of FM radio-based PCL. The results are shown in Fig.11 and Fig.12. In detail, Fig.11 shows the false alarm rate measured in the range band [150 $200] \mathrm{km}$ versus the nominal value while Fig. 12 reports the

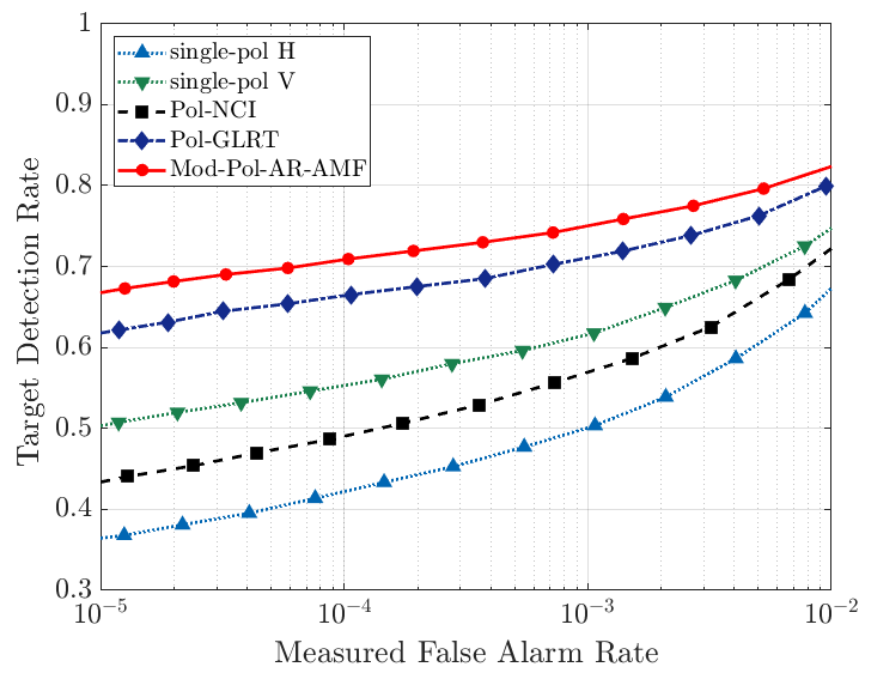

Fig.12 Empirical ROC curves with different polarimetric adaptive detection strategies for the bistatic range band [0-100] $\mathrm{km}$. 
empirical ROC curves. Note that the Mod-Pol-AR-AMF is applied with $Q=3$ and $J=200$ (S3). Incidentally, we recall that we measure the false alarm rate in the range band [150200] $\mathrm{km}$ to avoid labelling as false alarms detections corresponding to targets not identified by the available airtruth therefore enabling an unsupervised false alarm counting process.

From Fig.11 and Fig.12, the following comments apply:

i. The capability to control the false alarm rate changes with the employed detector. Specifically, the CA-CFAR detection scheme applied to the single-pol channel map yields the best capability to guarantee the nominal $P_{f a}$. In constrast, the CA-CFAR scheme applied after the Pol-NCI is the worst performing solution, confirming the weakness of the simplifying hypothesis of independent disturbance affecting the $L$ range-velocity maps. The false alarm rate control offered by the Mod-Pol-AR-AMF is comparable to that of the Pol-GLRT and, in both cases, largely acceptable in practical applications.

ii. The polarimetric approaches can effectively improve the performance of the system only if suitable strategies are employed to exploit signals collected via multi-polarized antennas. This is demonstrated by observing the performance of the Pol-NCI strategy. The latter, in fact, does not succed in improving the target detection performance with respect to the single $\mathrm{H}$ and $\mathrm{V}$ channels. This is due to the generally higher number of false alarms obtained, that confirms the unsuitability of this solution.

iii. The polarimetric strategies that adaptively exploit the information diversity to counteract the disturbance, namely the Pol-GLRT and the proposed Mod-Pol-ARAMF, substantially increase the target detection performance with respect to the single-pol channels and the Pol-NCI.

iv. The proposed Mod-Pol-AR-AMF solution is the best performing solution, yielding a tremendous improvement with respect to both the worst and best performing singlepol channel. Moreover, it further increases the capability to discriminate target of the previously proposed PolGLRT by approximately $7-8 \%$. This for example yields 461 additional correct detections at $P_{f a}=10^{-4}$.

The same considerations are confirmed observing Fig.13(a-c) where we show the raw detection results obtained for the same set of 50 consecutive data files considered in Fig.6 over the same bistatic range-velocity plane with nominal $P_{f a}=10^{-5}$. In detail, we report the results for the Pol-NCI, the Pol-GLRT and the Mod-Pol-AR-AMF in Fig.13(a),(b) and (c), respectively. We recall that the Mod-Pol-AR-AMF is now applied with the parameters and the adaptivity strategy selected after the careful tuning of Section IV. The benefits of this tuning are evident by comparing Fig.13(c) and Fig.6(c). In addition, the advantage of the Mod-Pol-AR-AMF is apparent with respect to both the alternative strategies that exploit polarization diversity in Fig.13 (a-b), see e.g. the target tracks at $[30-70] \mathrm{km}$ bistatic range and approx. $-300 \mathrm{~m} / \mathrm{s}$ bistatic velocity and at [100-150] $\mathrm{km}$ bistatic range and approx. $-400 \mathrm{~m} / \mathrm{s}$ bistatic velocity that are now detected with great continuity. Moreover, the improvement is tremendous with respect to the single-pol solutions in Fig.6(a-b).

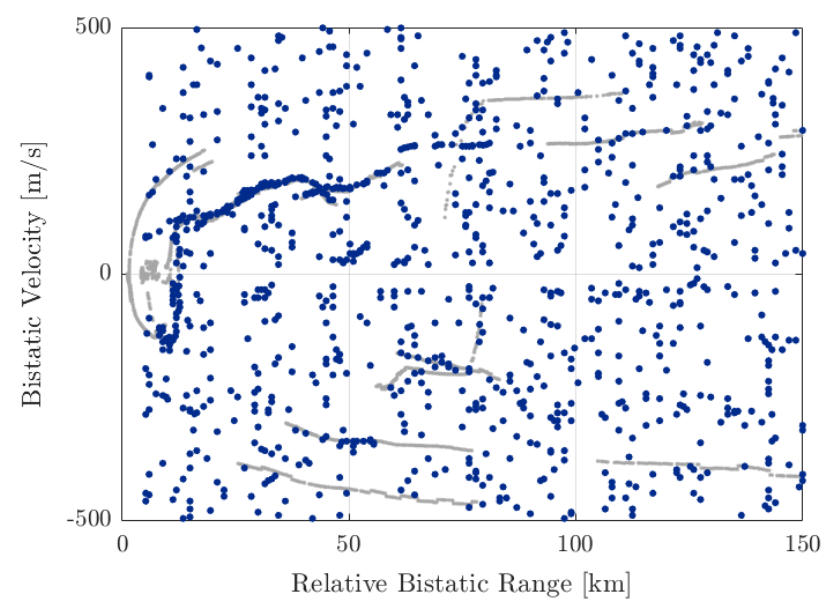

(a)

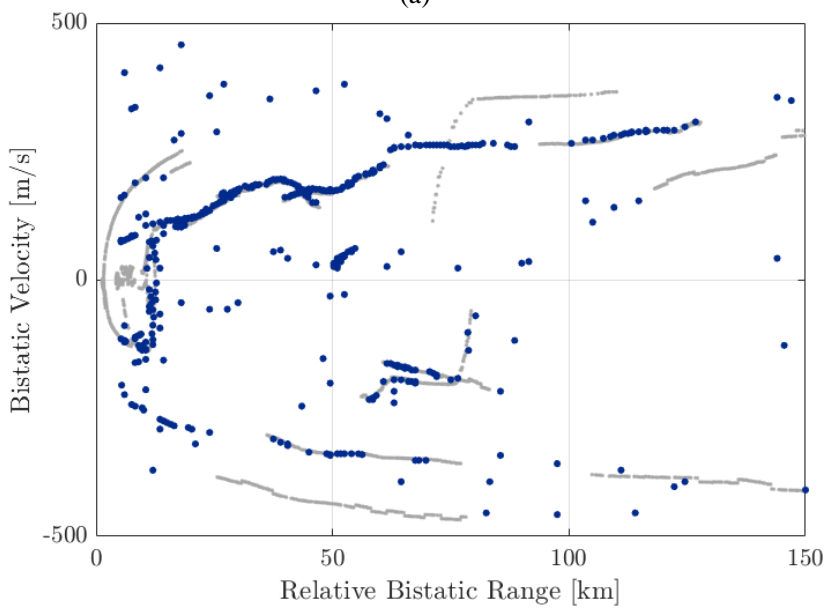

(b)

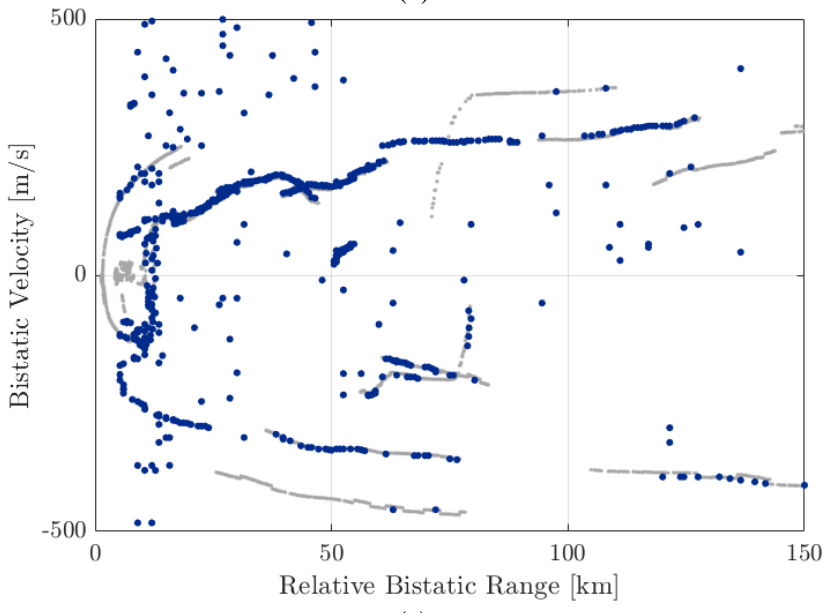

(c)

Fig.13 Detection results over 50 consecutive data files with $P_{f a}=10^{-5}$, using (a) Pol-NCI (b) Pol-GLRT

(c) Mod-Pol-AR-AMF, with $Q=3$ and $J=200$ (S3). 


\section{CONCLUSION}

In this paper, we presented an adaptive processing scheme to fruitfully exploit polarization diversity in PCL systems. The proposed strategy is based on a multichannel autoregressive disturbance model and leverages the information conveyed using multi-polarized surveillance channels. A cost-effective implementation of the proposed detector is introduced to reduce the computational burden while yielding negligible loss. The extensive experimental validation carried out against data collected using an FM radio-based PCL, demonstrates that the joint exploitation of polarimetric and temporal information according to the proposed strategy significantly improves the system performance. In fact, the derived parametric adaptive detector effectively rejects the disturbance and in turn significantly increases the target discrimination capability. This advantage is observed both with respect to the conventional single-pol processing scheme and with respect to previously proposed solutions to multipolarimetric detection in passive radar.

\section{APPENDIX}

In this Appendix we prove that, under the condition in (21), the asymptotic performance of the direct and costeffective implementations of the proposed detector are identical. We recall that the assumption in (21) is tantamount to write

$$
\boldsymbol{\Sigma}\left(m, \tau, f_{D}\right)=\boldsymbol{\Sigma}\left(0, \tau, f_{D}\right) e^{j 2 \pi m \frac{f_{D}}{f_{s}}}
$$

where $\boldsymbol{\Sigma}\left(0, \tau, f_{D}\right)=\left[\tilde{\mathbf{t}}\left(0, \tau, f_{D}\right) \otimes \mathbf{I}_{L}\right]$ being $\tilde{\mathbf{t}}\left(0, \tau, f_{D}\right)$ the vector of expected temporal returns for a unit amplitude target echo vector at the first sub-CPI, and it is exploited to provide the target echo energy focusing within the generic sub-CPI. The exponential term in (29) accounts for the Dopplerinduced phase compensation across subsequent overlapped sub-CPIs.

Accordingly, in the following we demonstrate that the asymptotic performance of the Mod-Pol-AR-AMF does not depend on $\tilde{\mathbf{t}}\left(0, \tau, f_{D}\right)$, or equivalently on $\boldsymbol{\Sigma}\left(0, \tau, f_{D}\right)$, provided that $\mathbf{F}=\mathbf{U}^{H} \boldsymbol{\Sigma}\left(0, \tau, f_{D}\right)$ is full-rank, being $\mathbf{U}$ the $L \times L Q$ matrix that realizes the singular value decomposition (SVD) of the normal matrix $\mathbf{P}$, i.e. $\mathbf{P}=\mathbf{U} \boldsymbol{\Lambda} \mathbf{U}^{H}$. This, in turn, shows that any approximation adopted for the vector $\tilde{\mathbf{t}}\left(0, \tau, f_{D}\right)$ that meets the above condition does not yield any performance degradation.

To this end, we first recall some results obtained by the authors in [15] and [16]. Note that the explicit dependency on the delay and Doppler bin $\left(\tau, f_{D}\right)$ will be omitted in the following to simplify the notation.

In [15] and [16], we have shown that, under the $H_{0}$ hypothesis and the assumption of perfectly known disturbance characteristics, vector $\overline{\mathbf{w}}_{0}=\left(\mathbf{D}_{w}^{-1 / 2}\right)^{H} \mathbf{w}_{0}$ is a complex Gaussian random variable with zero-mean vector and covariance matrix $\mathbf{I}_{L}$, i.e. $\left.\overline{\mathbf{w}}_{0}\right|_{H_{0}} \sim \mathcal{C N}\left(\mathbf{0}_{L \times 1}, \mathbf{I}_{L}\right)$.
Consequently, the distribution of the test statistic, scaled by a factor 2 , is a central Chi-squared distribution with $2 L$ degrees of freedom, i.e. $2\left\|\overline{\mathbf{w}}_{0}\right\|^{2} \sim \chi_{2 L}^{2}(0)$. Note that this result is independent of $\boldsymbol{\Sigma}(\mathrm{m})$ (see Appendix $\mathrm{C}$ of [15] for demonstration) and, in turn, the strategy adopted to set the detection threshold in order to achieve the desired $P_{f a}$ remains unaltered. In fact, the $P_{f a}$ expression is written as

$$
P_{f a}=\sum_{l=0}^{L-1} \frac{1}{\Gamma(L-l)}\left(\frac{\eta}{2}\right)^{l} e^{-\frac{\eta}{2}}
$$

where $\eta$ is the detection threshold. Consequently, if any loss arose from the use of an approximated vector $\tilde{\mathbf{t}}(0)$, see e.g. the approximation in (18), it should be attributed to the filter effect on the target returns rather than to a mismatch in term of disturbance cancellation.

In [15][16] we also derived the $P_{d}$ expressions, for both a non-fluctuating and fluctuating target model, under asymptotic conditions.

For a Swerling 0 target model [24], and assuming the disturbance parameters known, vector $\overline{\mathbf{w}}_{0}$ under the $H_{1}$ hypothesis is a complex Gaussian random vector, with mean vector $\quad \mathbf{v}=\left(\mathbf{D}_{w}{ }^{-\frac{1}{2}}\right)^{H} \sum_{m=0}^{M-Q} \boldsymbol{\Sigma}^{H}(m) \mathbf{P}[\tilde{\mathbf{t}}(m) \otimes \boldsymbol{\alpha}] \quad$ and covariance matrix $\mathbf{I}_{L}$, i.e. $\left.\overline{\mathbf{w}}_{0}\right|_{H_{1}} \sim \mathcal{C N}\left(\mathbf{v}, \mathbf{I}_{L}\right)$. Therefore, the asymptotic distribution of the test statistic, scaled by a factor 2 , is given by a non-central Chi-squared distribution with $2 L$ degrees of freedom and non-centrality parameter $\varsigma=\|\boldsymbol{v}\|^{2}$, i.e. $2\left\|\overline{\mathbf{w}}_{0}\right\|^{2} \sim \chi_{2 L}^{2}(\varsigma)$. Consequently, the asymptotic $P_{d}$ can be expressed using the Marcum $Q$-function, as follows

$$
\begin{aligned}
& P_{d}=Q_{L}(\sqrt{\varsigma}, \sqrt{\eta})= \\
& \int_{\sqrt{\eta}}^{\infty} x\left(\frac{x}{\sqrt{\varsigma}}\right)^{L-1} \exp \left(-\frac{x^{2}+\varsigma}{2}\right) I_{L-1}(\sqrt{\varsigma} x) d x
\end{aligned}
$$

where $I_{L-1}(\sqrt{\varsigma} x)$ denotes the modified Bessel function of order $L-1$.

We write the non-centrality parameter $\varsigma$ as

$$
\begin{aligned}
\varsigma=\sum_{m=0}^{M-Q}[\tilde{\mathbf{t}}(m) \otimes \boldsymbol{\alpha}]^{H} \mathbf{P} \boldsymbol{\Sigma}(m) \mathbf{D}_{w}^{-1} \\
\\
\times \sum_{n=0}^{M-Q} \boldsymbol{\Sigma}^{H}(n) \mathbf{P}[\tilde{\mathbf{t}}(n) \otimes \boldsymbol{\alpha}]
\end{aligned}
$$

We recall the definition of $\mathbf{D}_{w}$

$$
\mathbf{D}_{w}=\sum_{i=0}^{M-Q} \sum_{k=0}^{M-Q} \boldsymbol{\Sigma}^{H}(i) \mathbf{P} \mathbf{M}_{i k} \mathbf{P} \boldsymbol{\Sigma}(k)
$$


where $\mathbf{M}_{i k}=E\left\{\tilde{\mathbf{x}}_{0}(i) \tilde{\mathbf{x}}_{0}^{H}(k) \mid H_{0}\right\}$. By substituting (29) and (33) in (32), we can rework $\varsigma$ as follows

$$
\begin{gathered}
\varsigma=(M-Q+1)^{2}[\tilde{\mathbf{t}}(0) \otimes \boldsymbol{\alpha}]^{H} \mathbf{U} \boldsymbol{\Lambda} \mathbf{U}^{H} \boldsymbol{\Sigma}(0) \times \\
\left\{\boldsymbol{\Sigma}^{H}(0) \mathbf{U} \boldsymbol{\Lambda} \mathbf{U}^{H}\left[\sum_{i=0}^{M-Q} \sum_{k=0}^{M-Q} \mathbf{M}_{i k} e^{j 2 \pi \frac{f_{\boldsymbol{D}}}{f_{s}}(k-i)}\right] \mathbf{U} \boldsymbol{\Lambda} \mathbf{U}^{H} \boldsymbol{\Sigma}(0)\right\}^{-1} \\
\times \boldsymbol{\Sigma}^{H}(0) \mathbf{U} \boldsymbol{\Lambda} \mathbf{U}^{H}[\tilde{\mathbf{t}}(0) \otimes \boldsymbol{\alpha}]
\end{gathered}
$$

where we used the SVD of the $L$ rank matrix $\mathbf{P}=\mathbf{U} \boldsymbol{\Lambda} \mathbf{U}^{H}$.

Consequently, provided that the $L \times L$ matrix $\mathbf{F}=\mathbf{U}^{H} \boldsymbol{\Sigma}(0)$ is full-rank, eq. (34) is simplified as

$$
\begin{aligned}
\varsigma=(M-Q+1)^{2} & {[\tilde{\mathbf{t}}(0) \otimes \boldsymbol{\alpha}]^{H} \mathbf{U}\left(\mathbf{U}^{H} \mathbf{M}_{D} \mathbf{U}\right)^{-1} } \\
& \times \mathbf{U}^{H}[\tilde{\mathbf{t}}(0) \otimes \boldsymbol{\alpha}]
\end{aligned}
$$

where we defined $\mathbf{M}_{D}=\sum_{i=0}^{M-Q} \sum_{k=0}^{M-Q} \mathbf{M}_{i k} e^{j 2 \pi \frac{f_{D}}{f_{s}}(k-i)}$. Eq. (35) proves that the non-centrality parameter $\varsigma$ does not depend on the employed $\boldsymbol{\Sigma}(0)$, under the considered assumptions.

On the other hand, assuming a Swerling I target model [24], namely if the target complex amplitude is distributed as a zero-mean Gaussian random variable with covariance matrix $\mathbf{M}_{t}=E\left\{\boldsymbol{\alpha} \boldsymbol{\alpha}^{H}\right\}$, vector $\overline{\mathbf{w}}_{0}$ turns into a complex Gaussian random variable with zero-mean vector and covariance matrix $\mathbf{D}_{w}^{\prime}$.

Consequently, the asymptotic $P_{d}$ is given by

$$
P_{d}=\sum_{n=0}^{N-1} \sum_{k=0}^{\mu_{n}-1} \frac{-e^{-\frac{\eta}{\lambda_{n}}} \eta^{k}}{\Gamma(k+1)} \delta_{k, n}
$$

where $\lambda_{0} \ldots \lambda_{N-1}$ denote the $N \leq L$ distinct non-zero eigenvalues of $\mathbf{D}_{w}^{\prime}$, each with multiplicity $\mu_{n}$, and the coefficients $\delta_{k, n}, n=0, \ldots, N-1, k=0, \ldots, \mu_{n}-1$, can be evaluated using the formulas reported in [16]. We recall that matrix $\mathbf{D}_{w}^{\prime}$ is given by $\mathbf{D}_{w}^{\prime}=\mathbf{I}_{L}+\mathbf{Y}$, where $\mathbf{Y}$ can be written as follows, provided that eq. (21), and thus in (29), are valid

$$
\mathbf{Y}=(M-Q+1)^{2} \mathbf{D}_{w}^{-\frac{H}{2}}\left[\boldsymbol{\Sigma}^{H}(0) \mathbf{P} \mathbf{M}_{T} \mathbf{P} \boldsymbol{\Sigma}(0)\right] \mathbf{D}_{w}^{-\frac{1}{2}}
$$

with $\mathbf{M}_{T}=\tilde{\mathbf{t}}(0) \tilde{\mathbf{t}}^{H}(0) \otimes \mathbf{M}_{t}$.

By using the SVD decomposition for $\mathbf{P}$ and the definition of $\mathbf{F}$, we write $\mathbf{D}_{w}^{-\frac{1}{2}}$ as

$$
\mathbf{D}_{w}^{-\frac{1}{2}}=\mathbf{F}^{-1} \boldsymbol{\Lambda}^{-1}\left(\mathbf{U}^{H} \mathbf{M}_{D} \mathbf{U}\right)^{-\frac{1}{2}}
$$

Then, by substituting (38) into (37) we obtain

$$
\begin{aligned}
\mathbf{Y}=(M-Q+1)^{2} & \left(\mathbf{U}^{H} \mathbf{M}_{D} \mathbf{U}\right)^{-\frac{H}{2}}\left(\mathbf{U}^{H} \mathbf{M}_{T} \mathbf{U}\right) \\
& \times\left(\mathbf{U}^{H} \mathbf{M}_{D} \mathbf{U}\right)^{-\frac{1}{2}}
\end{aligned}
$$

Eq. (39) proves that matrix $\mathbf{Y}$, and therefore the eigenvalues of matrix $\mathbf{D}_{w}^{\prime}$, do not depend on the employed $\boldsymbol{\Sigma}(0)$ if this is selected so that matrix $\mathbf{F}$ is invertible.

Finally, we have demonstrated that, when eq. (21) holds, considering both a Swerling 0 and Swerling I target model, the asymptotic performance of the proposed detector does not change with the specific vector $\tilde{\mathbf{t}}\left(0, \tau, f_{D}\right)$ applied to the subCPI of $Q$ samples, provided that this is selected so that $\mathbf{F}=$ $\mathbf{U}^{H} \boldsymbol{\Sigma}(0)$ is full-rank. This is certainly the case for the approximation proposed in (18) that implies the neglect of the Doppler induced phase variation within the sub-CPI, so that it is concluded that it does not yield any loss in terms of target detection.

\section{REFERENCES}

[1] H. D. Griffiths and C. J. Baker, "Passive coherent location radar systems. Part 1: performance prediction," in IEE Proceedings - Radar, Sonar and Navigation, vol. 152, no. 3, pp. 153-159, 3 June 2005.

[2] C. J. Baker, H. D. Griffiths and I. Papoutsis, "Passive coherent location radar systems. Part 2: waveform properties," in IEE Proceedings Radar, Sonar and Navigation, vol. 152, no. 3, pp. 160-168, 3 June 2005.

[3] A. Farina, H. Kuschel, "IEEE Aerospace and Electronic Systems Magazine", Special Issue on Passive Radar Part I \& II, vol. 27, pp. 10$11,2012$.

[4] P. Lombardo and F. Colone, "Advanced processing methods for passive bistatic radar," in Melvin, W. L., and Scheer, J. A. (Eds.): 'Principles of Modern Radar: Advanced Radar Techniques', Raleigh, NC: SciTech Publishing, 2012, pp. 739-821.

[5] C. Bongioanni, F. Colone, T. Martelli, R. D'Angeli and P. Lombardo, "Exploiting polarimetric diversity to mitigate the effect of interferences in FM-based passive radar," 11-th INTERNATIONAL RADAR SYMPOSIUM, Vilnius, 2010, pp. 1-4.

[6] M. Conti, C. Moscardini, A. Capria, "Dual-polarization DVB-T passive radar: Experimental results", Proc. IEEE Radar Conf. 2016, pp. 1-5, May 2016.

[7] O. Cabrera, C. Bongioanni, F. Filippini, O.Sarabakha, F. Colone and P. Lombardo, "Detecting drones and human beings with DVB-S based COTS passive radar for short-range surveillance, " IEEE 2020 International Radar Conference, Washington DC, USA, 2020

[8] F. Colone and P. Lombardo: "Polarimetric passive coherent location", IEEE Transactions on Aerospace and Electronic Systems, 2015, 51, (2), pp. 1079-1097.

[9] F. Filippini, F. Colone, D. Cristallini and G. Bournaka, "Preliminary experimental results of polarimetric detection schemes for DVB-T based passive radar," 2017 IEEE Radar Conference (RadarConf), Seattle, WA, 2017, pp. 0383-0388.

[10] F. Filippini, F. Colone, D. Cristallini and G. Bournaka, "Experimental results of polarimetric detection schemes for DVB-T-based passive radar," in IET Radar, Sonar \& Navigation, vol. 11, no. 6, pp. 883-891, 62017.

[11] F. Filippini and F. Colone, "A practical approach to polarimetric adaptive target detection in passive radar," International Conference on Radar Systems (Radar 2017), Belfast, 2017, pp. 1-6.

[12] J. You, X. Wan, Y. Fu and G. Fang, "Experimental study of polarisation technique on multi-FM-based passive radar," in IET Radar, Sonar \& Navigation, vol. 9, no. 7, pp. 763-771, 82015. 
[13] F. Colone and P. Lombardo, "Non-coherent adaptive detection in passive radar exploiting polarimetric and frequency diversity," in IET Radar, Sonar \& Navigation, vol. 10, no. 1, pp. 15-23, 12016.

[14] I. Son and B. Yazici, "Passive polarimetric multistatic radar for ground moving target," 2016 IEEE Radar Conference (RadarConf), Philadelphia, PA, 2016, pp. 1-6.

[15] F.Colone and F.Filippini, "Auto-regressive model based polarimetric adaptive detection scheme Part I: theoretical derivation and performance analysis", IEEE Transactions on Aerospace and Electronic Systems, 2020.

[16] F.Colone and F.Filippini, "Auto-regressive model based polarimetric adaptive detection scheme Part II: performance assessment under spectral model mismatch", IEEE Transactions on Aerospace and Electronic Systems, 2020.

[17] F. Filippini, and F. Colone,: "Polarimetric Detection Scheme for Passive Radar based on a 2D Auto-Regressive Disturbance Model", 2019 International Radar Conference (RADAR), TOULON, France, 2019, pp. 1-6.

[18] K. Strøm et al., "DVB-T passive radar dual polarization measurements in the presence of strong direct signal interference," 2017 18th International Radar Symposium (IRS), Prague, 2017, pp. 1-9.
[19] Y. Yi, X. Wan, J. Yi and X. Cao, "Polarization Diversity Technology Research in Passive Radar Based on Subcarrier Processing," in IEEE Sensors Journal, vol. 19, no. 5, pp. 1710-1719, 1 March, 2019.

[20] X. Wang, X. Ai and H. Wang, "Experimental results of DTTB-based passive radar with polarisation diversity reception," in The Journal of Engineering, vol. 2019, no. 19, pp. 5677-5681, 102019.

[21] I. Pisciottano, D. Cristallini, D. Pastina and F. Santi, "Experimental Results of Polarimetric Passive ISAR Exploiting DVB-S2 Illumination," 2020 IEEE International Radar Conference (RADAR), Washington, DC, USA, 2020, pp. 518-523.

[22] F. Colone, C. Palmarini, T. Martelli and E. Tilli, "Sliding extensive cancellation algorithm for disturbance removal in passive radar," in IEEE Transactions on Aerospace and Electronic Systems, vol. 52, no. 3, pp. 1309-1326, June 2016

[23] K. J. Sohn, H. Li and B. Himed, "Parametric GLRT for Multichannel Adaptive Signal Detection," in IEEE Transactions on Signal Processing, vol. 55, no. 11, pp. 5351-5360, Nov. 2007

[24] P. Swerling, "Probability of detection for fluctuating targets," in IRE Trans. on Information Theory, vol. 6, no. 2, pp. 269-308, April 1960.

[25] A. Lauri, F. Colone, R. Cardinali, C. Bongioanni and P. Lombardo, "Analysis and Emulation of FM Radio Signals for Passive Radar," 2007 IEEE Aerospace Conference, Big Sky, MT, 2007, pp. 1-10.

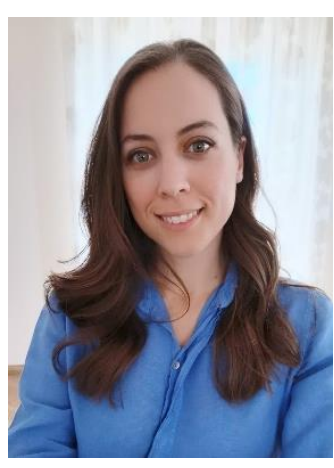

Francesca Filippini (S'17-M'20) received her M.Sc. degree (cum Laude) in Communication Engineering and the PhD degree in Radar and Remote Sensing, from Sapienza University of Rome, in 2016 and 2020, respectively. From January to May 2016, she has been working on her Master Thesis with the Passive Radar and Antijamming Techniques Department at Fraunhofer Institute FHR. She is currently a PostDoctoral Researcher with the Department of Information Engineering, Electronics and Telecommunications at Sapienza University of Rome.

Dr. Filippini received the 2018 Premium Award for the Best Paper in IET Radar, Sonar \& Navigation, the Best Paper Award at the 2019 Int. Radar Conference, the second Best Student Paper Award at the 2018 IEEE Radar Conference and the Best Paper Award at the 2017 GTTI Workshop on Radar and Remote Sensing. She is a Member of the IEEE Aerospace and Elecronic System Society (AESS) Board of Governors, where she is currently serving as Graduate Student Representative and Chair of the AESS Professional Networking and Mentoring Program.

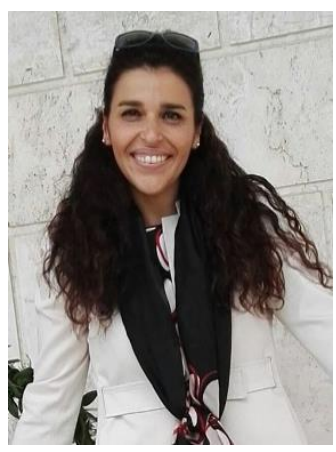

Fabiola Colone received the laurea degree (B.S.+M.S.) in Telecommunications Engineering and the Ph.D. degree in Remote Sensing from Sapienza University of Rome, Italy, in 2002 and 2006, respectively. She joined the DIET Dept. (formerly INFOCOM) of Sapienza University of Rome as a Research Associate in January 2006. From December 2006 to June 2007, she was a Visiting Scientist at the Electronic and Electrical Engineering Dept. of the University College London, London, U.K. She is currently an Associate Professor at the Faculty of Information Engineering, Informatics, and Statistics of Sapienza University of Rome.

The majority of Dr. Colone's research activity is devoted to radar systems and signal processing. She has been involved, with scientific responsibility roles, in research projects funded by the European Commission, the European Defence Agency, the Italian Space Agency, the Italian Ministry of Research, and the radar industry. Her research has been reported in over 120 publications in international technical journals, book chapters, and conference proceedings. Dr. Colone has been co-recipient of the 2018 Premium Award for Best Paper in IET Radar, Sonar \& Navigation.

Since 2017 she is member of the Board of Governors of the IEEE Aerospace and Electronic System Society (AESS) in which she is currently serving as Vice-President for Member Services, and Editor in Chief for the IEEE AESS QEB Newsletters. She is IEEE Senior Member from 2017 and member of the IEEE AESS Radar System Panel from 2019. Dr. Colone is Associate Editor for the IEEE Transactions on Signal Processing and member of the Editorial Board of the Int. Journal of Electronics and Communications (Elsevier). She served in the technical committee of many international conferences. She was in the organizing committee, as the Student Forum Co-Chair, of the IEEE 2008 Radar Conference (Rome, Italy), and she is currently in the organizing committee, as Special Sessions Co-Chair, of the IEEE 2020 Radar Conference (Florence, Italy). She is also Technical co-Chair of the IEEE Radar Conference 2021 (Atlanta, USA). 